\title{
Kondo Effect in the Presence of Spin-Orbit Coupling
}

\author{
Takashi Yanagisawa \\ Electronics and Photonics Research Institute, National Institute of Advanced Industrial Science \\ and Technology (AIST) Tsukuba Central 2, 1-1-1 Umezono, Tsukuba 305-8568, Japan
}

(Dated: Received April 8, 2012; published online September 4, 2012)

\begin{abstract}
Recently, a series of noncentrosymmetric superconductors has been a subject of considerable interest since the discovery of superconductivity in $\mathrm{CePt}_{3} \mathrm{Si}$. In noncentrosymmetric materials, the degeneracy of bands is lifted in the presence of spin-orbit coupling. This will bring about new effects in the Kondo effect since the band degeneracy plays an important role in the scattering of electrons by localized spins. We investigate the single-impurity Kondo problem in the presence of spin-orbit coupling. We examine the effect of spin-orbit coupling on the scattering of conduction electrons, by using the Green's function method, for the s-d Hamiltonian, with employing a decoupling procedure. As a result, we obtain a closed system of equations of Green's functions, from which we can calculate physical quantities. The Kondo temperature $T_{K}$ is estimated from a singularity of Green's functions. We show that $T_{K}$ is reduced as the spin-orbit coupling constant $\alpha$ is increased. When $2 \alpha k_{F}$ is comparable to or greater than $k_{B} T_{K}(\alpha=0), T_{K}$ shows an abrupt decrease as a result of the band splitting. This suggests a Kondo collapse accompanied with a sharp decrease of $T_{K}$. The $\log T$-dependence of the resistivity will be concealed by the spin-orbit interaction.
\end{abstract}

\section{INTRODUCTION}

The Kondo effect has attracted many researchers since the discovery of the solution of the resistance minimum 1 , 2]. The effect arises from the interactions between a single magnetic atom and the many electrons in a metal. Metals, when magnetic atoms are added, and rare earth compounds exhibit many interesting phenomena that are related to the Kondo effect. The spin-flip scattering of a conduction-electron spin by a localized impurity spin gives rise to a term proportional to $\ln T$ in the resistivity.

Superconductors without inversion symmetry have attracted much attention since the discovery of superconductivity in $\mathrm{CePt}_{3} \mathrm{Si}$ 3]. A group of noncentrosymmetric rare-earth compounds has been reported to exhibit superconductivity: for example, $\mathrm{Li}_{2} \mathrm{Pt}_{3} \mathrm{~B}$ [4, 5], $\mathrm{CeIrSi}_{3}$, $\mathrm{CeCoGe}_{3}, \mathrm{CeIrGe}_{3}[6]$, $]$, and $\mathrm{LaNiC}_{2}[9]$. The absence of spatial inversion yields the splitting of bands due to a spin-orbit interaction [10, 11].

The influence of the spin-orbit interaction was discussed very recently in two-dimensional systems starting from the single-impurity Anderson model [12 14]. In the conventional Kondo problem, the conduction-electron states with spin up and down are degenerate. We expect that the band splitting has a large effect on the Kondo effect, and is closely related to a multi-channel Kondo problem. The purpose of this paper is to investigate this subject on the basis of the s-d Hamiltonian with the spinorbit interaction of Rashba type in three dimensions at finite temperature. We calculate Green's functions and evaluate the Kondo temperature $T_{K}$ from a singularity of them. We show that $T_{K}$ is reduced as a result of the band splitting and shows a abrupt decrease when $\alpha k_{F}$ is comparable to $k_{B} T_{K}$.

The paper is organized as follows. In Section II we show the Hamiltonian, and in Section III we derive equations for Green's functions. We obtain an approximate solution in Section IV. The Kondo temperature and cor- rection to resistivity are discussed in subsequent Sections $\mathrm{V}$ and VI. In Section VII we examine the strong limit of the spin-orbit interaction where the details of calcuations are shown in Appexdix.

\section{MODEL HAMILTONIAN}

The Hamiltonian is $H=H_{0}+H_{s d}=H_{K}+H_{s o}+H_{s d}$ where

$$
\begin{aligned}
H_{K}= & \sum_{\mathbf{k}} \xi_{\mathbf{k}}\left(c_{\mathbf{k} \uparrow}^{\dagger} c_{\mathbf{k} \uparrow}+c_{\mathbf{k} \downarrow}^{\dagger} c_{\mathbf{k} \downarrow}\right) \\
H_{s o}= & \sum_{\mathbf{k}}\left[\alpha\left(i k_{x}+k_{y}\right) c_{\mathbf{k} \uparrow}^{\dagger} c_{\mathbf{k} \downarrow}+\alpha\left(-i k_{x}+k_{y}\right) c_{\mathbf{k} \downarrow}^{\dagger} c_{\mathbf{k} \uparrow}\right] \\
H_{s d}= & -\frac{J}{2} \frac{1}{N} \sum_{\mathbf{k k}^{\prime}}\left[S_{z}\left(c_{\mathbf{k} \uparrow}^{\dagger} c_{\mathbf{k}^{\prime} \uparrow}-c_{\mathbf{k} \downarrow}^{\dagger} c_{\mathbf{k}^{\prime} \downarrow}\right)+S_{+} c_{\mathbf{k} \downarrow}^{\dagger} c_{\mathbf{k}^{\prime} \uparrow}\right. \\
& \left.+S_{-} c_{\mathbf{k}_{\uparrow}}^{\dagger} c_{\mathbf{k}^{\prime} \downarrow}\right] .
\end{aligned}
$$

$\xi_{\mathbf{k}}$ is defined by $\xi_{\mathbf{k}}=\epsilon_{\mathbf{k}}-\mu$ where $\epsilon_{\mathbf{k}}$ is the dispersion relation of the conduction electrons and $\mu$ is the chemical potential. $c_{\mathbf{k} \sigma}$ and $c_{\mathbf{k} \sigma}^{\dagger}$ are annihilation and creation operators, respectively. We set $H_{0}=H_{K}+H_{s o} . S_{+}$, $S_{-}$and $S_{z}$ denote the operators of the localized spin. We consider the spin-orbit interaction of Rashba type in $H_{s o} . \alpha$ indicates the coupling constant of the spin-orbit interaction. The term $H_{s d}$ indicates the s-d interaction between the conduction electrons and the localized spin, with the coupling constant $J . J$ is negative for the antiferromagnetic interaction. 


\section{GREEN'S FUNCTIONS}

First, we define Green's functions of the conduction electrons

$$
\begin{aligned}
G_{\mathbf{k k}^{\prime} \sigma}(\tau) & =-\left\langle T_{\tau} c_{\mathbf{k} \sigma}(\tau) c_{\mathbf{k}^{\prime} \sigma}^{\dagger}(0)\right\rangle, \\
F_{\mathbf{k k}^{\prime}}(\tau) & =-\left\langle T_{\tau} c_{\mathbf{k} \downarrow}(\tau) c_{\mathbf{k}^{\prime} \uparrow}^{\dagger}(0)\right\rangle,
\end{aligned}
$$

where $T_{\tau}$ is the time ordering operator. We note that the spin operators satisfy the following relations:

$$
\begin{aligned}
S_{ \pm} S_{z} & =\mp \frac{1}{2} S_{z}, \quad S_{z} S_{ \pm}= \pm \frac{1}{2} S_{ \pm}, \\
S_{+} S_{-} & =\frac{3}{4}+S_{z}-S_{z}^{2}, \\
S_{-} S_{+} & =\frac{3}{4}-S_{z}-S_{z}^{2} .
\end{aligned}
$$

We also define Green's functions which include the localized spins as well as the conduction electron operators. They are for example, following the notation of Zubarev[15],

$$
\begin{aligned}
\left\langle\left\langle S_{z} c_{\mathbf{k} \uparrow} ; c_{\mathbf{k}^{\prime} \uparrow}^{\dagger}\right\rangle\right\rangle_{\tau}=-\left\langle T_{\tau} S_{z} c_{\mathbf{k} \uparrow}(\tau) c_{\mathbf{k}^{\prime} \uparrow}^{\dagger}(0)\right\rangle, \\
\left\langle\left\langle S_{-} c_{\mathbf{k}_{\downarrow}} ; c_{\mathbf{k}^{\prime} \uparrow}^{\dagger}\right\rangle\right\rangle_{\tau}=-\left\langle T_{\tau} S_{-} c_{\mathbf{k}_{\downarrow}}(\tau) c_{\mathbf{k}^{\prime} \uparrow}^{\dagger}(0)\right\rangle, \\
\left\langle\left\langle S_{z} c_{\mathbf{k}_{\downarrow}} ; c_{\mathbf{k}^{\prime} \uparrow}^{\dagger}\right\rangle\right\rangle_{\tau}=-\left\langle T_{\tau} S_{z} c_{\mathbf{k}_{\downarrow}}(\tau) c_{\mathbf{k}^{\prime} \uparrow}^{\dagger}(0)\right\rangle, \\
\left\langle\left\langle S_{-} c_{\mathbf{k} \uparrow} ; c_{\mathbf{k}^{\prime} \uparrow}^{\dagger}\right\rangle\right\rangle_{\tau}=-\left\langle T_{\tau} S_{-} c_{\mathbf{k} \uparrow}(\tau) c_{\mathbf{k}^{\prime} \uparrow}^{\dagger}(0)\right\rangle .
\end{aligned}
$$

The Fourier transforms are defined as usual:

$$
\begin{aligned}
G_{\mathbf{k k}^{\prime} \sigma}(\tau)= & \frac{1}{\beta} \sum_{n} e^{-i \omega_{n} \tau} G_{\mathbf{k k}^{\prime} \sigma}\left(i \omega_{n}\right) \\
F_{\mathbf{k k}^{\prime}}(\tau)= & \frac{1}{\beta} \sum_{n} e^{-i \omega_{n} \tau} F_{\mathbf{k k}^{\prime}}\left(i \omega_{n}\right) \\
\left\langle\left\langle S_{z} c_{\mathbf{k} \uparrow} ; c_{\mathbf{k}^{\prime} \uparrow}^{\dagger}\right\rangle\right\rangle_{\tau}= & \frac{1}{\beta} \sum_{n} e^{-i \omega_{n} \tau}\left\langle\left\langle S_{z} c_{\mathbf{k} \uparrow} ; c_{\mathbf{k}^{\prime} \uparrow}^{\dagger}\right\rangle\right\rangle_{i \omega_{n}} \\
& \ldots \ldots
\end{aligned}
$$

From the commutation relations

$$
\begin{aligned}
{\left[H_{0}, c_{\mathbf{k} \uparrow}\right] } & =-\xi_{\mathbf{k}} c_{\mathbf{k} \uparrow}-\alpha\left(i k_{x}+k_{y}\right) c_{\mathbf{k} \downarrow}, \\
{\left[H_{s d}, c_{\mathbf{k} \uparrow}\right] } & =-\frac{J}{2 N} \sum_{\mathbf{k}^{\prime}}\left(-S_{z} c_{\mathbf{k}^{\prime} \uparrow}-S_{-} c_{\mathbf{k}^{\prime} \downarrow}\right)
\end{aligned}
$$

the equation of motion for $G_{\mathbf{k k}^{\prime} \uparrow}(\tau)$ reads

$$
\begin{aligned}
\frac{\partial}{\partial \tau} G_{\mathbf{k k}^{\prime} \uparrow}(\tau)= & -\delta(\tau) \delta_{\mathbf{k k}^{\prime}}-\xi_{\mathbf{k}} G_{\mathbf{k k}^{\prime} \uparrow}(\tau) \\
& -\alpha\left(i k_{x}+k_{y}\right) F_{\mathbf{k k}^{\prime}}(\tau) \\
+ & \frac{J}{2 N} \sum_{\mathbf{q}}\left[\left\langle\left\langle S_{z} c_{\mathbf{q} \uparrow} ; c_{\mathbf{k}^{\prime} \uparrow}^{\dagger}\right\rangle\right\rangle_{\tau}+\left\langle\left\langle S_{-} c_{q \downarrow} ; c_{\mathbf{k}^{\prime} \uparrow}^{\dagger}\right\rangle\right\rangle_{\tau}\right] .
\end{aligned}
$$

Similarly, the equation of motion for $F_{\mathbf{k k}^{\prime}}$ is

$$
\begin{aligned}
\frac{\partial}{\partial \tau} F_{\mathbf{k k}^{\prime}}(\tau) & =-\xi_{\mathbf{k}} F_{\mathbf{k k}^{\prime}}-\alpha\left(-i k_{x}+k_{y}\right) G_{\mathbf{k k}^{\prime} \uparrow}(\tau) \\
& -\frac{J}{2 N} \sum_{q}\left[\left\langle\left\langle S_{z} c_{\mathbf{q} \downarrow} ; c_{\mathbf{k}^{\prime} \uparrow}^{\dagger}\right\rangle\right\rangle_{\tau}-\left\langle\left\langle S_{+} c_{\mathbf{q} \uparrow} ; c_{\mathbf{k}^{\prime} \uparrow}^{\dagger}\right\rangle\right\rangle_{\tau}\right] .
\end{aligned}
$$

We define

$$
\begin{aligned}
\Gamma_{\mathbf{k k}^{\prime}}(\tau) & =\frac{1}{\beta} \sum_{n} e^{-i \omega_{n}} \Gamma_{\mathbf{k k}^{\prime}}\left(i \omega_{n}\right) \\
& =\left\langle\left\langle S_{z} c_{\mathbf{k} \uparrow} ; c_{\mathbf{k}^{\prime} \uparrow}^{\dagger}\right\rangle\right\rangle_{\tau}+\left\langle\left\langle S_{-} c_{\mathbf{k} \downarrow} ; c_{\mathbf{k}^{\prime} \uparrow}^{\dagger}\right\rangle\right\rangle_{\tau}, \\
\Phi_{\mathbf{q k}}(\tau) & =\frac{1}{\beta} \sum_{n} e^{-i \omega_{n} \tau} \Phi_{\mathbf{q k}}\left(i \omega_{n}\right) \\
& =\left\langle\left\langle S_{z} c_{\mathbf{q} \downarrow}-S_{+} c_{\mathbf{q} \uparrow} ; c_{\mathbf{k} \uparrow}^{\dagger}\right\rangle,\right.
\end{aligned}
$$

then we obtain

$$
\begin{aligned}
\left(i \omega_{n}-\xi_{\mathbf{k}}\right) G_{\mathbf{k k}^{\prime} \uparrow}\left(i \omega_{n}\right) & =\delta_{\mathbf{k k}^{\prime}}+\alpha\left(i k_{x}+k_{y}\right) F_{\mathbf{k k}^{\prime}}\left(i \omega_{n}\right) \\
& -\frac{J}{2 N} \sum_{\mathbf{q k}^{\prime}} \Gamma_{\mathbf{q k}^{\prime}}\left(i \omega_{n}\right) \\
\left(i \omega_{n}-\xi_{\mathbf{k}}\right) F_{\mathbf{k k}^{\prime}}\left(i \omega_{n}\right) & =\alpha\left(-i k_{x}+k_{y}\right) G_{\mathbf{k k}^{\prime} \uparrow}\left(i \omega_{n}\right) \\
& +\frac{J}{2 N} \sum_{q} \Phi_{\mathbf{q k}^{\prime}}\left(i \omega_{n}\right)
\end{aligned}
$$

To obtain the solution to the equations above, we need Green's functions in eqs.(9)-(12). The equations of motion for $\left\langle\left\langle S_{z} c_{\mathbf{k} \uparrow} ; c_{\mathbf{k}^{\prime} \uparrow}^{\dagger}\right\rangle\right\rangle$ and $\left\langle\left\langle S_{-} c_{\mathbf{k}_{\downarrow}} ; c_{\mathbf{k}^{\prime} \uparrow}^{\dagger}\right\rangle\right\rangle$ are

$$
\begin{aligned}
& \left(i \omega-\xi_{\mathbf{k}}\right)\left\langle\left\langle S_{z} c_{\mathbf{k} \uparrow} ; c_{\mathbf{k}^{\prime} \uparrow}^{\dagger}\right\rangle\right\rangle_{i \omega} \\
= & \left\langle S_{z}\right\rangle \delta_{\mathbf{k}^{\prime}}+\alpha\left(i k_{x}+k_{y}\right)\left\langle\left\langle S_{z} c_{\mathbf{k} \downarrow} ; c_{\mathbf{k}^{\prime} \uparrow}^{\dagger}\right\rangle\right\rangle_{i \omega} \\
- & \frac{J}{2 N} \sum_{\mathbf{q}}\left[\left\langle\left\langle S_{z}^{2} c_{\mathbf{q} \uparrow} ; c_{\mathbf{k}^{\prime} \uparrow}^{\dagger}\right\rangle\right\rangle_{i \omega}+\frac{1}{2}\left\langle\left\langle S_{-} c_{\mathbf{q} \downarrow} ; c_{\mathbf{k}^{\prime} \uparrow}^{\dagger}\right\rangle\right\rangle_{i \omega}\right] \\
- & \frac{J}{2 N} \sum_{\mathbf{q q}^{\prime}}\left[\left\langle\left\langle S_{+} c_{\mathbf{k} \uparrow} c_{\mathbf{q}_{\downarrow}}^{\dagger} c_{\mathbf{q}^{\prime} \uparrow} ; c_{\mathbf{k}^{\prime} \uparrow}^{\dagger}\right\rangle\right\rangle_{i \omega}\right. \\
- & \left.\left\langle\left\langle S_{-} c_{\mathbf{k} \uparrow} c_{\mathbf{q}^{\prime} \uparrow}^{\dagger} c_{\mathbf{q}^{\prime} \downarrow} ; c_{\mathbf{k}^{\prime} \uparrow}^{\dagger}\right\rangle\right\rangle_{i \omega}\right],
\end{aligned}
$$

$$
\begin{aligned}
& \left(i \omega-\xi_{\mathbf{k}}\right)\left\langle\left\langle S_{-} c_{\mathbf{k} \downarrow} ; c_{\mathbf{k}^{\prime} \uparrow}^{\dagger}\right\rangle\right\rangle_{i \omega} \\
= & \alpha\left(-i k_{x}+k_{y}\right)\left\langle\left\langle S_{-} c_{\mathbf{k}_{\uparrow}} ; c_{\mathbf{k}^{\prime} \uparrow}^{\dagger}\right\rangle\right\rangle_{i \omega} \\
- & \frac{J}{4 N} \sum_{\mathbf{q}^{\prime}}\left\langle\left\langle S_{-} c_{\mathbf{q}^{\prime} \downarrow} ; c_{\mathbf{k}^{\prime} \uparrow}^{\dagger}\right\rangle\right\rangle_{i \omega} \\
+ & \frac{J}{2 N} \sum_{\mathbf{q}^{\prime}}\left[\left\langle\left\langle S_{-} c_{\mathbf{k}_{\downarrow} \downarrow} c_{\mathbf{q}_{\uparrow}}^{\dagger} c_{\mathbf{q}^{\prime} \uparrow} ; c_{\mathbf{k}^{\prime} \uparrow}^{\dagger}\right\rangle\right\rangle_{i \omega}\right. \\
& \left.-\left\langle\left\langle S_{-} c_{\mathbf{k}_{\downarrow} \downarrow} c_{\mathbf{q}_{\downarrow}}^{\dagger} c_{\mathbf{q}^{\prime} \downarrow} ; c_{\mathbf{k}^{\prime} \uparrow}^{\dagger}\right\rangle\right\rangle_{i \omega}-2\left\langle\left\langle S_{z} c_{\mathbf{k} \downarrow} c_{\mathbf{q}_{\downarrow}}^{\dagger} c_{\mathbf{q}^{\prime} \uparrow} ; c_{\mathbf{k}^{\prime} \uparrow}^{\dagger}\right\rangle\right\rangle_{i \omega}\right] \\
& -\frac{J}{2 N} \sum_{\mathbf{q}^{\prime}}\left\langle\left\langle S_{+} S_{-} c_{\mathbf{q}^{\prime} \uparrow} ; c_{\mathbf{k}^{\prime} \uparrow}^{\dagger}\right\rangle\right\rangle_{i \omega} .
\end{aligned}
$$


We use the commutation relation $S_{+} S_{-}=3 / 4+S_{z}-S_{z}^{2}$ to obtain

$$
\begin{aligned}
& \left(i \omega-\xi_{\mathbf{k}}\right) \Gamma_{\mathbf{k k}^{\prime}}(i \omega) \\
= & \delta_{\mathbf{k k}^{\prime}}\left\langle S_{z}\right\rangle+\alpha\left(i k_{x}+k_{y}\right)\left\langle\left\langle S_{z} c_{\mathbf{k}_{\downarrow}} ; c_{\mathbf{k}^{\prime} \uparrow}^{\dagger}\right\rangle\right\rangle_{i \omega} \\
+ & \alpha\left(-i k_{x}+k_{y}\right)\left\langle\left\langle S_{-} c_{\mathbf{k} \uparrow} ; c_{\mathbf{k}^{\prime} \uparrow}^{\dagger}\right\rangle\right\rangle_{i \omega} \\
- & \frac{J}{2 N} \sum_{\mathbf{q}}\left[\frac{3}{4}\left\langle\left\langle c_{\mathbf{q} \uparrow} ; c_{\mathbf{k}^{\prime} \uparrow}^{\dagger}\right\rangle\right\rangle_{i \omega}+\Gamma_{\mathbf{q k}^{\prime}}(i \omega)\right] \\
- & \frac{J}{2 N} \sum_{\mathbf{q q}^{\prime}}\left[\left\langle\left\langle S_{+} c_{\mathbf{k} \uparrow} c_{\mathbf{q}_{\downarrow}}^{\dagger} c_{\mathbf{q}^{\prime} \uparrow} ; c_{\mathbf{k}^{\prime} \uparrow}^{\dagger}\right\rangle\right\rangle_{i \omega}\right. \\
- & \left\langle\left\langle S_{-} c_{\mathbf{k}_{\uparrow} \uparrow} c_{\mathbf{q}_{\uparrow} \uparrow}^{\dagger} c_{\mathbf{q}^{\prime} \downarrow} ; c_{\mathbf{k}^{\prime} \uparrow}^{\dagger}\right\rangle\right\rangle_{i \omega}-\left\langle\left\langle S_{-} c_{\mathbf{k}_{\downarrow} \downarrow} c_{\mathbf{q}_{\uparrow}}^{\dagger} c_{\mathbf{q}^{\prime} \uparrow} ; c_{\mathbf{k}^{\prime} \uparrow}^{\dagger}\right\rangle\right\rangle_{i \omega} \\
+ & \left\langle\left\langle S_{-} c_{\mathbf{k}_{\downarrow} \downarrow} c_{\mathbf{q}_{\downarrow} \downarrow}^{\dagger} c_{\mathbf{q}^{\prime} \downarrow} ; c_{\mathbf{k}^{\prime} \uparrow}^{\dagger}\right\rangle\right\rangle_{i \omega} \\
+ & \left.2\left\langle\left\langle S_{z} c_{\mathbf{k} \downarrow} c_{\mathbf{q}_{\downarrow}}^{\dagger} c_{\mathbf{q}^{\prime} \uparrow} ; c_{\mathbf{k}^{\prime} \uparrow}^{\dagger}\right\rangle\right\rangle_{i \omega}\right] .
\end{aligned}
$$

Here we assume that $\left\langle S_{z}\right\rangle=0$. Now we need $\left\langle\left\langle S_{z} c_{\mathbf{k} \downarrow} ; c_{\mathbf{k}^{\prime} \uparrow}^{\dagger}\right\rangle\right\rangle$ and $\left\langle\left\langle S_{-} c_{\mathbf{k} \uparrow} ; c_{\mathbf{k}^{\prime} \uparrow}^{\dagger}\right\rangle\right\rangle$ to obtain a solution for $\Gamma_{\mathbf{k k}^{\prime}}$. The equations for $\left\langle\left\langle S_{z} c_{\mathbf{k} \downarrow} ; c_{\mathbf{k}^{\prime} \uparrow}^{\dagger}\right\rangle\right\rangle$ and $\left\langle\left\langle S_{-} c_{\mathbf{k} \uparrow} ; c_{\mathbf{k}^{\uparrow} \uparrow}^{\dagger}\right\rangle\right\rangle \operatorname{read}$

$$
\begin{aligned}
& \left(i \omega-\xi_{\mathbf{k}}\right)\left\langle\left\langle S_{z} c_{\mathbf{k} \downarrow} ; c_{\mathbf{k}^{\prime} \uparrow}^{\dagger}\right\rangle\right\rangle_{i \omega} \\
& =\alpha\left(-i k_{x}+k_{y}\right)\left\langle\left\langle S_{z} c_{\mathbf{k} \downarrow} ; c_{\mathbf{k}^{\prime} \uparrow}^{\dagger}\right\rangle\right\rangle_{i \omega} \\
& +\frac{J}{2 N} \sum_{q}\left[\left\langle\left\langle S_{z}^{2} c_{\mathbf{q} \downarrow} ; c_{\mathbf{k}^{\prime} \uparrow}^{\dagger}\right\rangle\right\rangle_{i \omega}-\frac{1}{2}\left\langle\left\langle S_{+} c_{\mathbf{q} \uparrow} ; c_{\mathbf{k}^{\prime} \uparrow}^{\dagger}\right\rangle\right\rangle_{i \omega}\right] \\
& -\frac{J}{2 N} \sum_{\mathbf{q q}^{\prime}}\left[\left\langle\left\langle S_{+} c_{\mathbf{q}_{\downarrow}}^{\dagger} c_{\mathbf{q}^{\prime} \downarrow} c_{\mathbf{k} \downarrow} ; c_{\mathbf{k}^{\prime} \uparrow}^{\dagger}\right\rangle\right\rangle_{i \omega}\right. \\
& \left.-\left\langle\left\langle S_{-} c_{\mathbf{q} \uparrow}^{\dagger} c_{\mathbf{q}^{\prime} \downarrow} c_{\mathbf{k} \downarrow} ; c_{\mathbf{k}^{\prime} \uparrow}^{\dagger}\right\rangle\right\rangle_{i \omega}\right],
\end{aligned}
$$

$$
\begin{aligned}
& \left(i \omega-\xi_{\mathbf{k}}\right)\left\langle\left\langle S_{-} c_{\mathbf{k} \uparrow} ; c_{\mathbf{k}^{\prime} \uparrow}^{\dagger}\right\rangle\right\rangle_{i \omega} \\
= & -\delta_{\mathbf{k k}^{\prime}}\left\langle S_{-}\right\rangle+\alpha\left(i k_{x}+k_{y}\right)\left\langle\left\langle S_{-} c_{\mathbf{k} \downarrow} ; c_{\mathbf{k}^{\prime} \uparrow}^{\dagger}\right\rangle\right\rangle_{i \omega} \\
+ & \frac{J}{4 N} \sum_{\mathbf{q}^{\prime}}\left\langle\left\langle S_{-} c_{\mathbf{q}^{\prime} \uparrow} ; c_{\mathbf{k}^{\prime} \uparrow}^{\dagger}\right\rangle\right\rangle_{i \omega} \\
+ & \frac{J}{2 N} \sum_{\mathbf{q q}^{\prime}}\left[\left\langle\left\langle S_{-} c_{\mathbf{k} \uparrow} c_{\mathbf{q}_{\downarrow}}^{\dagger} c_{\mathbf{q}^{\prime} \downarrow} ; c_{\mathbf{k}^{\prime} \uparrow}^{\dagger}\right\rangle\right\rangle_{i \omega}\right. \\
- & \left.\left\langle\left\langle S_{-} c_{\mathbf{k} \uparrow} c_{\mathbf{q} \uparrow}^{\dagger} c_{\mathbf{q}^{\prime} \uparrow} ; c_{\mathbf{k}^{\prime} \uparrow}^{\dagger}\right\rangle\right\rangle_{i \omega}+2\left\langle\left\langle S_{z} c_{\mathbf{q}_{\downarrow}}^{\dagger} c_{\mathbf{q}^{\prime} \downarrow} c_{\mathbf{k} \uparrow} ; c_{\mathbf{k}^{\prime} \uparrow}^{\dagger}\right\rangle\right\rangle_{i \omega}\right] .
\end{aligned}
$$

\section{APPROXIMATE SOLUTION}

We assume that the spin-orbit coupling $\alpha$ is small and we keep terms up to the order of $\alpha$. We adopt the ap- proximation that

$$
\begin{aligned}
\left\langle\left\langle S_{z} c_{\mathbf{k} \downarrow} ; c_{\mathbf{k}^{\prime} \uparrow}^{\dagger}\right\rangle\right\rangle_{i \omega}=\frac{\alpha\left(-i k_{x}+k_{y}\right)}{i \omega-\xi_{\mathbf{k}}}\left\langle\left\langle S_{z} c_{\mathbf{k} \uparrow} ; c_{\mathbf{k}^{\prime} \uparrow}^{\dagger}\right\rangle\right\rangle_{i \omega}, \\
\left\langle\left\langle S_{-} c_{\mathbf{k} \uparrow} ; c_{\mathbf{k}^{\prime} \uparrow}^{\dagger}\right\rangle\right\rangle_{i \omega}=\frac{\alpha\left(i k_{x}+k_{y}\right)}{i \omega-\xi_{\mathbf{k}}}\left\langle\left\langle S_{-} c_{\mathbf{k} \downarrow} ; c_{\mathbf{k}^{\prime} \uparrow}^{\dagger}\right\rangle\right\rangle_{i \omega} \cdot(31)
\end{aligned}
$$

This means that we have neglected the terms of the order of $J \alpha$ in the right-hand side. Then we obtain

$$
\begin{aligned}
& \left(i \omega-\xi_{\mathbf{k}}-\frac{\alpha^{2}\left(k_{x}^{2}+k_{y}^{2}\right)}{i \omega-\xi_{\mathbf{k}}}\right) \Gamma_{\mathbf{k k}^{\prime}}(i \omega) \\
= & -\frac{J}{2 N} \sum_{\mathbf{q}}\left[\frac{3}{4}\left\langle\left\langle c_{\mathbf{q} \uparrow} ; c_{\mathbf{k}^{\prime} \uparrow}^{\dagger}\right\rangle\right\rangle_{i \omega}+\Gamma_{\mathbf{q k}^{\prime}}(i \omega)\right] \\
- & \frac{J}{2 N} \sum_{\mathbf{q} \mathbf{q}^{\prime}}\left[\left\langle\left\langle S_{+} c_{\mathbf{k} \uparrow} c_{\mathbf{q} \downarrow}^{\dagger} c_{\mathbf{q}^{\prime} \uparrow} ; c_{\mathbf{k}^{\prime} \uparrow}^{\dagger}\right\rangle\right\rangle_{i \omega}\right. \\
- & \left\langle\left\langle S_{-} c_{\mathbf{k} \uparrow} c_{\mathbf{q}_{\downarrow}}^{\dagger} c_{\mathbf{q}^{\prime} \downarrow} ; c_{\mathbf{k}^{\prime} \uparrow}^{\dagger}\right\rangle\right\rangle_{i \omega}-\left\langle\left\langle S_{-} c_{\mathbf{k}_{\downarrow}} c_{\mathbf{q}_{\uparrow} \uparrow}^{\dagger} c_{\mathbf{q}^{\prime} \uparrow} ; c_{\mathbf{k}^{\prime} \uparrow}^{\dagger}\right\rangle\right\rangle_{i \omega} \\
+ & \left.\left\langle\left\langle S_{-} c_{\mathbf{k} \downarrow} c_{\mathbf{q}_{\downarrow}}^{\dagger} c_{\mathbf{q}^{\prime} \downarrow} ; c_{\mathbf{k}^{\prime} \uparrow}^{\dagger}\right\rangle\right\rangle_{i \omega}+2\left\langle\left\langle S_{z} c_{\mathbf{k} \downarrow} c_{\mathbf{q}_{\downarrow} \downarrow}^{\dagger} c_{\mathbf{q}^{\prime} \downarrow} ; c_{\mathbf{k}^{\prime} \uparrow}^{\dagger}\right\rangle\right\rangle_{i \omega}\right]
\end{aligned}
$$

We use the same approximation in the right-hand side of eq. (23), that is, $\left(i \omega-\xi_{\mathbf{k}}\right) F_{\mathbf{k k}^{\prime}}(i \omega)=\alpha\left(-i k_{x}+\right.$ $\left.k_{y}\right) G_{\mathbf{k k}^{\prime} \uparrow}(i \omega)$, and we have

$$
\begin{aligned}
\left(i \omega-\xi_{\mathbf{k}}\right) G_{\mathbf{k k}^{\prime} \uparrow}(i \omega) & =\delta_{\mathbf{k k}^{\prime}}-\frac{J}{2 N} \sum_{\mathbf{p}} \Gamma_{\mathbf{p k}^{\prime}}(i \omega) \\
& +\frac{\alpha^{2}\left(k_{x}^{2}+k_{y}^{2}\right)}{i \omega-\xi_{\mathbf{k}}} G_{\mathbf{k k}^{\prime} \uparrow}(i \omega)
\end{aligned}
$$

Here, we employ the decoupling approximation procedure for Green's functions [16, 17]. Many-body Green's functions are approximated as follows.

$$
\begin{aligned}
\left\langle\left\langle S_{-} c_{\mathbf{k} \uparrow} c_{\mathbf{q}^{\prime} \uparrow}^{\dagger} c_{\mathbf{q}^{\prime} \downarrow} ; c_{\mathbf{k}^{\prime} \uparrow}^{\dagger}\right\rangle\right\rangle_{i \omega} & \approx\left\langle c_{\mathbf{k} \uparrow} c_{\mathbf{q}^{\prime} \uparrow}^{\dagger}\right\rangle\left\langle\left\langle S_{-} c_{\mathbf{q}^{\prime} \downarrow} ; c_{\mathbf{k}^{\prime} \uparrow}^{\dagger}\right\rangle\right\rangle_{i \omega} \\
& +\left\langle S_{-} c_{\mathbf{q}^{\prime} \uparrow}^{\dagger} c_{\mathbf{q}^{\prime} \downarrow}\right\rangle\left\langle\left\langle c_{\mathbf{k} \uparrow} ; c_{\mathbf{k}^{\prime} \uparrow}^{\dagger}\right\rangle\right\rangle_{i \omega}, \\
\left\langle\left\langle S_{+} c_{\mathbf{k} \uparrow} c_{\mathbf{q}_{\downarrow}}^{\dagger} c_{\mathbf{q}^{\prime} \uparrow} ; c_{\mathbf{k}^{\prime} \uparrow}^{\dagger}\right\rangle\right\rangle_{i \omega} & \approx\left\langle S_{+} c_{\mathbf{q}_{\downarrow}}^{\dagger} c_{\mathbf{q}^{\prime} \uparrow}\right\rangle\left\langle\left\langle c_{\mathbf{k} \uparrow} ; c_{\mathbf{k}^{\prime} \uparrow}^{\dagger}\right\rangle\right\rangle_{i \omega} \\
& -\left\langle S_{+} c_{\mathbf{q}_{\downarrow}}^{\dagger} c_{\mathbf{k}^{\prime} \uparrow}\right\rangle\left\langle\left\langle c_{\mathbf{q}^{\prime} \uparrow} ; c_{\mathbf{k}^{\prime} \uparrow}^{\dagger}\right\rangle\right\rangle_{i \omega},
\end{aligned}
$$

$$
\begin{aligned}
\left\langle\left\langle S_{z} c_{\mathbf{k} \downarrow} c_{\mathbf{q}_{\downarrow}}^{\dagger} c_{\mathbf{q}^{\prime} \uparrow} ; c_{\mathbf{k}^{\prime} \uparrow}^{\dagger}\right\rangle\right\rangle_{i \omega} & \approx\left\langle c_{\mathbf{k} \downarrow} c_{\mathbf{q}_{\downarrow}}^{\dagger}\right\rangle\left\langle\left\langle S_{z} c_{\mathbf{q}^{\prime} \uparrow} ; c_{\mathbf{k}^{\prime} \uparrow}^{\dagger}\right\rangle\right\rangle_{i \omega} \\
& -\left\langle S_{z} c_{\mathbf{q}_{\downarrow}}^{\dagger} c_{\mathbf{k} \downarrow}\right\rangle\left\langle\left\langle c_{\mathbf{q}^{\prime} \uparrow} ; c_{\mathbf{k}^{\prime} \uparrow}^{\dagger}\right\rangle\right\rangle_{i \omega},
\end{aligned}
$$

$$
\begin{aligned}
\left\langle\left\langle S_{-} c_{\mathbf{k} \downarrow} c_{\mathbf{q}_{\downarrow}}^{\dagger} c_{\mathbf{q}^{\prime} \downarrow} ; c_{\mathbf{k}^{\prime} \uparrow}^{\dagger}\right\rangle\right\rangle_{i \omega} & \approx\left\langle c_{\mathbf{q} \downarrow} c_{\mathbf{q}^{\prime} \downarrow}^{\dagger}\right\rangle\left\langle\left\langle S_{-} c_{\mathbf{k}_{\downarrow}} ; c_{\mathbf{k}^{\prime} \uparrow}^{\dagger}\right\rangle\right\rangle_{i \omega} \\
& +\left\langle c_{\mathbf{k} \downarrow} c_{\mathbf{q}_{\downarrow}}^{\dagger}\right\rangle\left\langle\left\langle S_{-} c_{\mathbf{q}^{\prime} \downarrow} ; c_{\mathbf{k}^{\prime} \uparrow}^{\dagger}\right\rangle\right\rangle_{i \omega} .
\end{aligned}
$$


We define

$$
\begin{aligned}
& n_{\mathbf{k}}=\sum_{\mathbf{q}}\left\langle c_{\mathbf{q} \uparrow}^{\dagger} c_{\mathbf{k} \uparrow}\right\rangle=\sum_{\mathbf{q}}\left\langle c_{\mathbf{q} \downarrow}^{\dagger} c_{\mathbf{k} \downarrow}\right\rangle \\
& m_{k}=3 \sum_{\mathbf{q}}\left\langle S_{-} c_{\mathbf{q} \uparrow}^{\dagger} c_{\mathbf{k} \downarrow}\right\rangle=2 \sum_{\mathbf{q}}\left(\left\langle S_{z} c_{\mathbf{q} \uparrow}^{\dagger} c_{\mathbf{k} \uparrow}\right\rangle+\left\langle S_{-} c_{\mathbf{q} \uparrow}^{\dagger} c_{\mathbf{k} \downarrow}\right\rangle\right) .
\end{aligned}
$$

We used the relation obtained from the rotational symmetry in the spin space,

$$
\left\langle S_{-} c_{\mathbf{q}_{\uparrow}}^{\dagger} c_{\mathbf{q}^{\prime} \downarrow}\right\rangle=\left\langle S_{+} c_{\mathbf{q}_{\downarrow}}^{\dagger} c_{\mathbf{q}^{\prime} \uparrow}\right\rangle=2\left\langle S_{z} c_{\mathbf{q}_{\uparrow}}^{\dagger} c_{\mathbf{q}^{\prime} \uparrow}\right\rangle=-2\left\langle S_{z} c_{\mathbf{q}_{\downarrow}}^{\dagger} c_{\mathbf{q}^{\prime} \downarrow}\right\rangle .
$$

Then, after the analytic continuation $i \omega \rightarrow \omega+i \delta$, we have

$$
\begin{gathered}
\left(\omega-\xi_{\mathbf{k}}-\frac{\alpha^{2} k_{\perp}^{2}}{\omega-\xi_{\mathbf{k}}}\right) \Gamma_{\mathbf{k k}^{\prime}}(\omega)+\left(\frac{3}{4}-m_{\mathbf{k}}\right) \\
\times \frac{J}{2 N} \sum_{\mathbf{q}} G_{\mathbf{q k}^{\prime}}(\omega)+\left(n_{\mathbf{k}}-\frac{1}{2}\right) \frac{J}{N} \sum_{\mathbf{q}} \Gamma_{\mathbf{q k}^{\prime}}(\omega)=0 \\
\left(\omega-\xi_{\mathbf{k}}-\frac{\alpha^{2} k_{\perp}^{2}}{\omega-\xi_{\mathbf{k}}}\right) G_{\mathbf{k k}^{\prime}}(\omega)+\frac{J}{2 N} \sum_{\mathbf{q}} \Gamma_{\mathbf{q k}^{\prime}}(\omega)=\delta_{\mathbf{k k}^{\prime}}
\end{gathered}
$$

where we set $k_{\perp}^{2}=k_{x}^{2}+k_{y}^{2}$. Then, we obtain from eqs.(41) and (42) that

$$
\begin{aligned}
\Gamma_{\mathbf{k k}^{\prime}}(\omega) & =G_{\mathbf{k}}^{0}(\omega)\left(m_{\mathbf{k}}-\frac{3}{4}\right) \frac{J}{2 N} G_{\mathbf{k}^{\prime}}^{0}(\omega) \\
& -G_{\mathbf{k}}^{0}(\omega)\left[\left(n_{\mathbf{k}}-\frac{1}{2}\right) J+\left(m_{\mathbf{k}}-\frac{3}{4}\right)\left(\frac{J}{2}\right)^{2} F(\omega)\right] \\
& \times \frac{1}{N} \sum_{\mathbf{q}} \Gamma_{\mathbf{q k}}(\omega)
\end{aligned}
$$

where

$$
\begin{aligned}
G_{\mathbf{k}}^{0}(\omega) & =\frac{1}{2}\left(\frac{1}{\omega-\xi_{\mathbf{k}}+\alpha k_{\perp}}+\frac{1}{\omega-\xi_{\mathbf{k}}-\alpha k_{\perp}}\right) \\
F(\omega) & =\frac{1}{N} \sum_{\mathbf{k}} G_{\mathbf{k}}^{0}(\omega) \\
G(\omega) & =\frac{1}{N} \sum_{\mathbf{k}}\left(n_{\mathbf{k}}-\frac{1}{2}\right) G_{\mathbf{k}}^{0}(\omega) \\
\Gamma(\omega) & =\frac{1}{N} \sum_{\mathbf{k}}\left(m_{\mathbf{k}}-\frac{3}{4}\right) G_{\mathbf{k}}^{0}(\omega) .
\end{aligned}
$$

Because of

$$
\frac{1}{N} \sum_{\mathbf{q}} \Gamma_{\mathbf{q k}}(\omega)=\frac{J}{2 N} \Gamma(\omega) G_{\mathbf{k}}^{0}(\omega) \frac{1}{1+J G(\omega)+(J / 2)^{2} \Gamma(\omega) F(\omega)}, \times \frac{1}{4}\left(\tanh \left(\frac{\xi_{\mathbf{k}}-\alpha k_{\perp}}{2 T_{K}}\right)+\tanh \left(\frac{\xi_{\mathbf{k}}+\alpha k_{\perp}}{2 T_{K}}\right)\right)=0,
$$

we obtain

$$
\begin{aligned}
\Gamma_{\mathbf{k k}^{\prime}}(\omega) & =\frac{J}{2 N} G_{\mathbf{k}}^{0}(\omega) G_{\mathbf{k}^{\prime}}^{0}(\omega)\left[\left(m_{\mathbf{k}}-\frac{3}{4}\right)(1+J G(\omega))\right. \\
& \left.-\left(n_{\mathbf{k}}-\frac{1}{2}\right) J \Gamma(\omega)\right] \\
& \times \frac{1}{1+J G(\omega)+(J / 2)^{2} \Gamma(\omega) F(\omega)} \\
G_{\mathbf{k k}^{\prime}}(\omega) & =\delta_{\mathbf{k k}^{\prime}} G_{\mathbf{k}}^{0}(\omega)-\frac{J^{2}}{4 N} \Gamma(\omega) G_{\mathbf{k}}^{0}(\omega) G_{\mathbf{k}^{\prime}}^{0}(\omega) \\
& \times \frac{1}{1+J G(\omega)+(J / 2)^{2} \Gamma(\omega) F(\omega)} \\
& =\delta_{\mathbf{k k}^{\prime}} G_{\mathbf{k}}^{0}(\omega)+\frac{J}{N} G_{\mathbf{k}}^{0}(\omega) G_{\mathbf{k}^{\prime}}^{0}(\omega) t(\omega),
\end{aligned}
$$

where we defined

$$
t(\omega)=-\frac{J}{4} \frac{\Gamma(\omega)}{1+J G(\omega)+(J / 2)^{2} \Gamma(\omega) F(\omega)} .
$$

$m_{\mathbf{k}}$ is given by

$$
\begin{aligned}
m_{\mathbf{k}}^{*} & =2 \sum_{\mathbf{q}}\left(\left\langle S_{z} c_{\mathbf{k} \uparrow}^{\dagger} c_{\mathbf{q} \uparrow}\right\rangle\left\langle S_{-} c_{\mathbf{k} \uparrow}^{\dagger} c_{\mathbf{q} \downarrow}\right\rangle\right)=2 \sum_{\mathbf{q}} \Gamma_{\mathbf{q k}}(\tau=-0) \\
& =\frac{2}{\beta} \sum_{\mathbf{q} \omega_{n}} e^{i \omega_{n} \delta} \Gamma_{\mathbf{q k}}\left(i \omega_{n}\right)
\end{aligned}
$$

where $\delta$ is an infinitesimal constant. Because $m_{\mathbf{k}}$ is real, we obtain

$$
m_{\mathbf{k}}=-4 \frac{1}{\beta} \sum_{\omega_{n}} e^{i \omega_{n} \delta} G_{\mathbf{k}}^{0}\left(i \omega_{n}\right) t\left(i \omega_{n}\right)
$$

Similarly we have

$$
n_{\mathbf{k}}=\frac{1}{\beta} \sum_{\omega_{n}} G_{\mathbf{k}}^{0}\left(i \omega_{n}\right)\left(1+J F\left(i \omega_{n}\right) t\left(i \omega_{n}\right)\right) .
$$

\section{KONDO TEMPERATURE}

A singularity of $t(\omega)$ determines the characteristic temperature of the system. We investigate the hightemperature region where $m_{\mathbf{k}}=0$. Then,

$$
\Gamma(\omega)=-\frac{3}{4} F(\omega) \text {. }
$$

We obtain for $\mathbf{k}=\mathbf{k}^{\prime}$

$$
G_{\mathbf{k k}}(\omega)^{-1}=G_{\mathbf{k}}^{0}(\omega)^{-1}-\frac{3 J^{2}}{16 N} \frac{F(\omega)}{1+J G(\omega)}+O\left(J^{4}\right)
$$

The Kondo temperature $T_{K}$ is determined by the vanishing of the denominator in this equation:

$$
1-J \frac{1}{2 N} \sum_{\mathbf{k}}\left(\frac{1}{\omega-\xi_{\mathbf{k}}+\alpha k_{\perp}}+\frac{1}{\omega-\xi_{\mathbf{k}}-\alpha k_{\perp}}\right)
$$


where

$$
\xi_{\mathbf{k}}=\frac{1}{2 m}\left(k_{\perp}^{2}+k_{z}^{2}\right)-\mu .
$$

We have used $n_{\mathbf{k}}=\left(f\left(\xi_{k}-\alpha k_{\perp}\right)+f\left(\xi_{k}+\alpha k_{\perp}\right)\right) / 2$ by neglecting the term of the order of $J^{2}$. By using the expansion,

$$
\tanh \left(\frac{z}{2}\right)=\sum_{n=-\infty}^{\infty} \frac{1}{z-i \pi(2 n+1)}
$$

the equation for $T_{K}$ is

$$
\begin{aligned}
1 & =J \sum_{n=-\infty}^{\infty} \frac{1}{8(2 \pi)^{2}} \sqrt{\frac{2 m}{\mu}} \int_{0}^{K} d k_{\perp} k_{\perp}\left[2 \frac{i \pi T_{K} \operatorname{sign}(2 n+1)}{\omega-i \pi(2 n+1) T_{K}}\right. \\
& +\frac{i \pi T_{K} \operatorname{sign}(2 n+1)}{\omega+2 \alpha k_{\perp}-i \pi(2 n+1) T_{K}} \\
& \left.+\frac{i \pi T_{K} \operatorname{sign}(2 n+1)}{\omega-2 \alpha k_{\perp}-i \pi(2 n+1) T_{K}}\right]
\end{aligned}
$$

where $K$ is a cutoff and we use an approximate expression

$$
\begin{aligned}
& \int_{-K}^{K} d k_{z} \frac{1}{\omega-k_{\perp}^{2} /(2 m)+\alpha k_{\perp}-k_{z}^{2} /(2 m)+\mu} \\
\times & \frac{1}{k_{\perp}^{2} /(2 m)+k_{z}^{2} /(2 m)+\alpha k_{\perp}-\mu-i \pi(2 n+1) T_{K}} \\
\approx & \sqrt{\frac{2 m}{\mu}} i \pi \operatorname{sign}(2 n+1) T_{K} \frac{1}{\omega+2 \alpha k_{\perp}-i \pi(2 n+1) T_{K}} .
\end{aligned}
$$

We set an cutoff $n_{0} \equiv D /\left(2 \pi T_{K}\right)$ in the summation with respect to $n$. By using the formula for the digamma function,

$$
\sum_{n=0}^{n_{0}} \frac{1}{n+\frac{1}{2}+x}=\psi\left(\frac{1}{2}+x+n_{0}\right)-\psi\left(\frac{1}{2}+x\right),
$$

we obtain

$$
\begin{aligned}
1 & =|J| \frac{1}{32 \pi^{2}} \sqrt{\frac{2 m}{\mu}} \int_{0}^{K} d k_{\perp} k_{\perp}\left[4 \log \left(\frac{2 e^{\gamma} D}{\pi T_{K}}\right)+2 \psi\left(\frac{1}{2}\right)\right. \\
& -\frac{1}{2} \psi\left(\frac{1}{2}-\frac{\omega+2 \alpha k_{\perp}}{i 2 \pi T_{K}}\right) \\
& -\frac{1}{2} \psi\left(\frac{1}{2}+\frac{\omega+2 \alpha k_{\perp}}{i 2 \pi T_{K}}\right)-\frac{1}{2} \psi\left(\frac{1}{2}-\frac{\omega-2 \alpha k_{\perp}}{i 2 \pi T_{K}}\right) \\
& \left.-\frac{1}{2} \psi\left(\frac{1}{2}+\frac{\omega-2 \alpha k_{\perp}}{i 2 \pi T_{K}}\right)\right] .
\end{aligned}
$$

We set $\mu=k_{F}^{2} /(2 m)$ and $K=2 k_{F}$, and expand the digamma function in terms of $\alpha k_{\perp} /\left(2 \pi T_{K}\right)$. For $\omega=0$, we have

$$
\begin{aligned}
1 & =|J| \frac{m k_{F}}{2 \pi^{2}}\left[\log \left(\frac{2 e^{\gamma} D}{\pi T_{K}}\right)-\frac{7 \zeta(3)}{2 \pi^{2}}\left(\frac{2 \alpha k_{F}}{T_{K}}\right)^{2}\right. \\
& \left.+\frac{31 \zeta(5)}{12 \pi^{4}}\left(\frac{2 \alpha k_{F}}{T_{K}}\right)^{4}-\ldots\right] .
\end{aligned}
$$

This yields the temperature $T_{K}$ as

$$
\begin{aligned}
k_{B} T_{K} & =\frac{2 e^{\gamma} D}{\pi} \exp \left[-\frac{1}{\rho_{F}|J|}-\frac{7 \zeta(3)}{2 \pi^{2}}\left(\frac{2 \alpha k_{F}}{k_{B} T_{K}}\right)^{2}\right. \\
& \left.+\frac{31 \zeta(5)}{12 \pi^{4}}\left(\frac{2 \alpha k_{F}}{k_{B} T_{K}}\right)^{4}-\cdots\right]
\end{aligned}
$$

where we introduced the Boltzmann constant $k_{B}$ and the density of states $\rho_{F}=m k_{F} /\left(2 \pi^{2}\right)$. This is a selfconsistency equation for $T_{K}$, and yields

$$
\begin{aligned}
x & =\exp \left[-0.21314\left(\frac{\alpha_{r}}{x}\right)^{2}+0.0550\left(\frac{\alpha_{r}}{x}\right)^{4}\right. \\
& -0.01655\left(\frac{\alpha_{r}}{x}\right)^{6}+0.005396\left(\frac{\alpha_{r}}{x}\right)^{8}-0.001822\left(\frac{\alpha_{r}}{x}\right)^{10} \\
& +\cdots] \\
& \equiv g(x),
\end{aligned}
$$

with variables

$$
x=T_{K} / T_{K}^{0}, \quad \alpha_{r}=2 \alpha k_{F} / k_{B} T_{K}^{0},
$$

where

$$
k_{B} T_{K}^{0}=\frac{2 e^{\gamma} D}{\pi} \exp \left(-\frac{1}{\rho_{F}|J|}\right) .
$$

We expanded $g(x)$ in powers of $\alpha_{r} / x$ up to the tenth order. The function $g(x)$ is shown in Fig.1, where higherorder terms are small and negligible except near $x \sim$ 0 . The equation $x=g(x)$ has no finite solution when $\alpha_{r}>1.045$. This indicates that $T_{K}$ vanishes when the spin-orbit coupling $\alpha k_{F}$ is greater than $1.045 k_{B} T_{K}^{0}$, and indicates a Kondo collapse with a sharp decrease of $T_{K}$. This may overestimate the reduction of $T_{K}$. When $\alpha$ is very large, if we use the asymptotic relation $\psi(1 / 2+z) \sim$ $\log (z)$, we obtain

$$
1 \simeq \rho_{F}|J|\left[\log \left(\frac{2 e^{\gamma} D}{\pi k_{B} T}\right)-\frac{1}{2} \log \left(\frac{2 \alpha k_{F}}{\pi k_{B} T}\right)+\frac{1}{4}\right] .
$$

This yields

$$
k_{B} T_{K} \simeq \frac{\sqrt{e}}{2 \alpha k_{F}} \frac{\left(2 e^{\gamma} D\right)^{2}}{\pi} \exp \left(-\frac{2}{\rho_{F}|J|}\right)=\frac{\pi \sqrt{e}}{\alpha_{r}} k_{B} T_{K}^{0},
$$

for $\alpha_{r} \gg 1$. We show $T_{K}$ as a function of $\alpha_{r}$ in Fig.2.

We expect that,in the strong coupling limit $\alpha_{r} \gg 1$, $T_{K}$ should approach that of single-band model:

$$
k_{B} T_{K}^{\alpha}=\frac{2 e^{\gamma} D}{\pi} \exp \left(-\frac{2}{\rho_{F}|J|}\right) .
$$

We will show this in the section 7 . This agrees with eq.(70) for $\alpha k_{F} \sim D$. This is very small compared to the original $T_{K}$ because $T_{K}^{\alpha} / T_{K}^{0} \simeq k_{B} T_{K}^{0} / D$. Therefore $T_{K}$ decreases as $\alpha_{r}$ is increased and shows up a sharp decrease at $\alpha_{r} \sim 1$. 


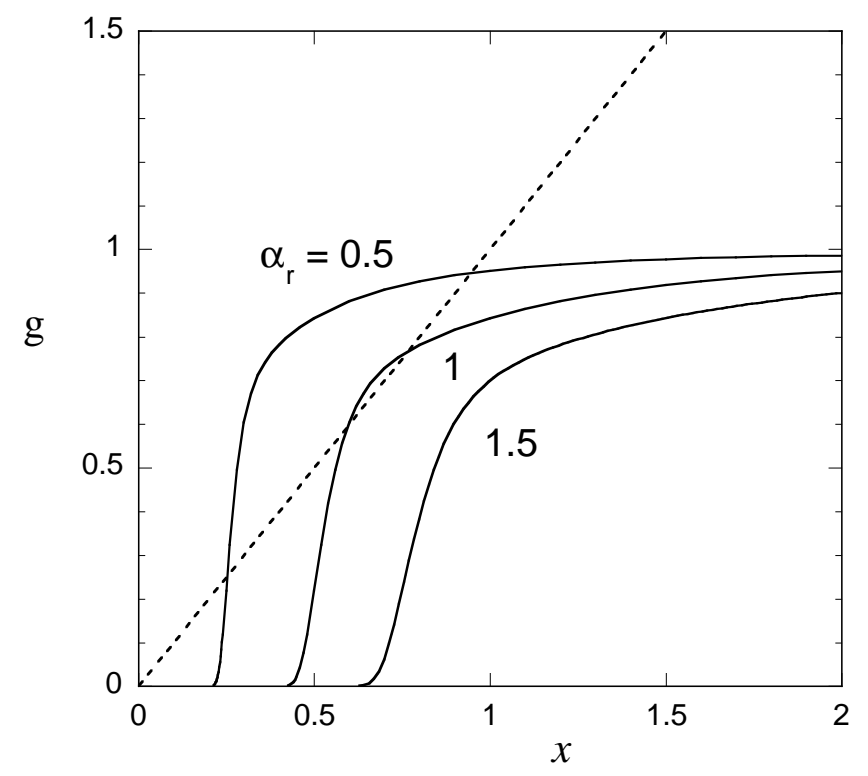

FIG. 1: $\quad g(x)=\exp \left(-\left(7 \zeta(3) / 2 \pi^{2}\right)\left(\alpha_{r} / x\right)^{2}+\cdots\right)$ up to the tenth order of $\alpha_{r} / x$ as a function of $x$ for $\alpha_{r}=0.5,1$ and 1.5. The straight line is a linear function $x$.

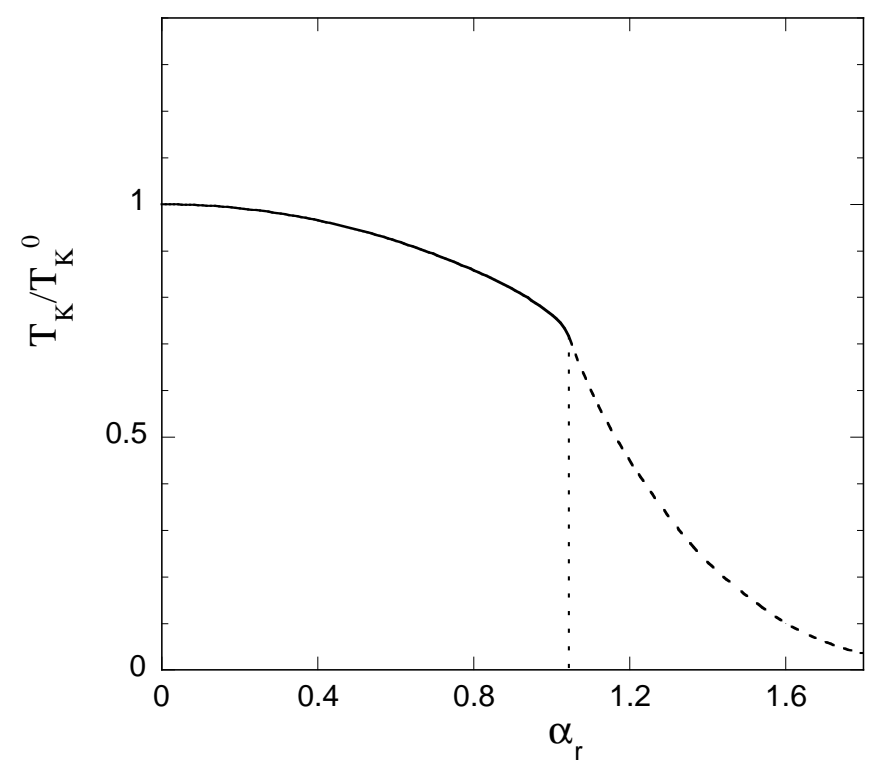

FIG. 2: $T_{K}$ as a function of $\alpha_{r} . x=g(x)$ has no solution for $\alpha_{r}>1.045$ (Kondo collapse) as indicated by dotted line. The dashed line is an expected line.

\section{CORRECTION TO RESISTIVITY}

The imaginary part of $G_{\mathbf{k k}}(\omega)^{-1}$ gives the scattering rate of conduction electrons due to the localized spin.
The inverse of the life time $\tau_{k}(\omega)$ is

$$
\begin{aligned}
\frac{1}{\tau_{k}(\omega)} & =n_{i} N \operatorname{Im} G_{\mathbf{k k}}(\omega)^{-1} \\
& =\frac{3 n_{i} J^{2}}{16} \pi \rho^{\alpha}(\omega) \frac{1+J K(\omega)}{(1+J K(\omega))^{2}+(J L(\omega))^{2}},
\end{aligned}
$$

where $n_{i}$ is the concentration of magnetic impurities. We have defined $K(\omega)=\operatorname{Re} G(\omega+i \delta), L(\omega)=-\operatorname{Im} G(\omega+i \delta)$, and $\rho^{\alpha}(\omega)=-(1 / \pi) \operatorname{Im} F(\omega+i \delta)$. Because we obtain

$$
K(0) \simeq \rho_{F}\left[\log \left(\frac{2 e^{\gamma} D}{\pi k_{B} T}\right)-\frac{7 \zeta(3)}{4 \pi^{2}}\left(\frac{2 \alpha k_{F}}{k_{B} T}\right)^{2}\right],
$$

the formula of the conductivity yields

$$
\begin{aligned}
\sigma & =-\frac{2 e^{2}}{3} \int \tau_{k}\left(\xi_{k}\right) v_{k}^{2} \frac{\partial f}{\partial \xi_{k}} \rho\left(\xi_{k}\right) \mathrm{d} \xi_{k} \\
& \simeq \frac{2 e^{2}}{3} v_{F}^{2} \rho(0) \frac{16}{3 \pi n_{i} J^{2} \rho^{\alpha}(0)}(1-|J| K(0)) \\
& \simeq \frac{2 e^{2}}{3} v_{F}^{2} \rho_{F} \frac{16}{3 \pi n_{i}|J|} \frac{\rho_{F}}{\rho^{\alpha}(0)}\left[\log \left(\frac{T}{T_{K}^{0}}\right)\right. \\
& \left.+\frac{7 \zeta(3)}{4 \pi^{2}}\left(\frac{2 \alpha k_{F}}{k_{B} T}\right)^{2}\right]
\end{aligned}
$$

We have the term $(\alpha / T)^{2}$ that comes from the spin-orbit interaction, and this term will conceal the logarithmic dependence of the resistivity. Then, the electrical resistivity $R$ in the high temperature region $T \gg T_{K}^{0}$ is

$$
\begin{aligned}
R & =R_{0} \\
& \times\left[1-|J| \rho_{F} \log \left(\frac{2 e^{\gamma} D}{\pi k_{B} T}\right)+\frac{7 \zeta(3)}{4 \pi^{2}}|J| \rho_{F}\left(\frac{2 \alpha k_{F}}{k_{B} T}\right)^{2}\right]^{-1}
\end{aligned}
$$

where $R_{0}$ is a constant. If the term $\left(\alpha / k_{B} T\right)^{2}$ is larger than the logarithmic term, the resistivity even shows $R \sim T^{2}$. Hence, the spin-orbit coupling may change the temperature dependence of the resistivity drastically.

\section{STRONG SPIN-ORBIT COUPLING CASE}

In this section let us consider the case with strong spin-orbit coupling. For this purpose, we diagonalize the Hamiltonian $H_{0}$ :

$$
\begin{aligned}
H_{0} & =\sum_{\mathbf{k}}\left(c_{\mathbf{k} \uparrow}^{\dagger} c_{\mathbf{k} \downarrow}^{\dagger}\right)\left(\begin{array}{cc}
\xi_{\mathbf{k}} & \alpha\left(i k_{x}+k_{y}\right) \\
\alpha\left(-i k_{x}+k_{y}\right) & \xi_{\mathbf{k}}
\end{array}\right)\left(\begin{array}{c}
c_{\mathbf{k} \uparrow} \\
c_{\mathbf{k} \downarrow}
\end{array}\right) \\
& =\sum_{\mathbf{k}}\left[\left(\xi_{\mathbf{k}}-\alpha k_{\perp}\right) \alpha_{\mathbf{k}}^{\dagger} \alpha_{\mathbf{k}}+\left(\xi_{\mathbf{k}}+\alpha k_{\perp}\right) \beta_{\mathbf{k}}^{\dagger} \beta_{\mathbf{k}}\right]
\end{aligned}
$$

where $k_{\perp}=\sqrt{k_{x}^{2}+k_{y}^{2}}$, and $\alpha_{\mathbf{k}}$ and $\beta_{\mathbf{k}}$ are defined by

$$
\begin{aligned}
\alpha_{\mathbf{k}} & =u_{\mathbf{k}} c_{\mathbf{k} \uparrow}+v_{\mathbf{k}} c_{\mathbf{k} \downarrow}, \\
\beta_{\mathbf{k}} & =-v_{\mathbf{k}}^{*} c_{\mathbf{k} \uparrow}+u_{\mathbf{k}} c_{\mathbf{k} \downarrow} .
\end{aligned}
$$


The coefficients $u_{\mathbf{k}}$ and $v_{\mathbf{k}}$ are

$$
u_{\mathbf{k}}=\frac{1}{2}, \quad v_{\mathbf{k}}=-\frac{i k_{x}+k_{y}}{\sqrt{2} k_{\perp}}
$$

satisfying $u_{\mathbf{k}}^{2}+\left|v_{\mathbf{k}}\right|^{2}=1$. We consider the case where the band split is so large that we can neglect the upper band. This means that we keep terms that contain $\alpha$-operators only. In this approximation the s-d interaction term is

$$
\begin{aligned}
H_{s d}^{\alpha} & =-\frac{J}{2} \frac{1}{N} \sum_{\mathbf{k k}^{\prime}}\left[S _ { z } \left\{\left(u_{k} u_{k^{\prime}}-v_{k} v_{k^{\prime}}^{*}\right) \alpha_{\mathbf{k}}^{\dagger} \alpha_{\mathbf{k}^{\prime}}\right.\right. \\
& \left.+S_{+} v_{k} u_{k^{\prime}} \alpha_{\mathbf{k}}^{\dagger} \alpha_{\mathbf{k}^{\prime}}+S_{-} u_{k} v_{k^{\prime}}^{*} \alpha_{\mathbf{k}}^{\dagger} \alpha_{\mathbf{k}^{\prime}}\right]
\end{aligned}
$$

This is the model of one-channel conduction-electron band that interacts with the localized spin.

Let us consider the following Green's function:

$$
G_{\mathbf{k k}^{\prime}}^{\alpha}(\tau)=-\left\langle T_{\tau} \alpha_{\mathbf{k}}(\tau) \alpha_{\mathbf{k}^{\prime}}^{\dagger}(0)\right\rangle
$$

By using the same method in previous sections, $G_{k k^{\prime}}^{\alpha}$ is shown to be

$$
G_{\mathbf{k k}^{\prime}}^{\alpha}(z)=\frac{\delta_{\mathbf{k k}^{\prime}}}{z-\xi_{\mathbf{k} \alpha}}+\frac{J}{2 N} \frac{\frac{1}{2}+v_{\mathbf{k}} v_{\mathbf{k}^{\prime}}^{*}}{\left(z-\xi_{\mathbf{k} \alpha}\right)\left(z-\xi_{\mathbf{k}^{\prime} \alpha}\right)} t(z),
$$

for arbitrary complex number $z$ where we defined

$$
\begin{gathered}
t(z)=\frac{3 J}{16} \frac{F_{\alpha}(z)}{1+\frac{J}{2} G_{\alpha}(z)-\frac{3}{16}\left(\frac{J}{2}\right)^{2} F_{\alpha}(z)^{2}}, \\
F_{\alpha}(z)=\frac{1}{N} \sum_{\mathbf{k}} \frac{1}{z-\xi_{\mathbf{k} \alpha}} \\
G_{\alpha}(z)=\frac{1}{N} \sum_{\mathbf{k}} \frac{\bar{n}_{\mathbf{k} \alpha}-1 / 2}{z-\xi_{\mathbf{k} \alpha}} .
\end{gathered}
$$

We derive this formula in Appendix. The Kondo temperature $T_{K}^{\alpha}$ is determined from a singularity of $t(z)$ in the same way as previous sections. We obtain

$$
k_{B} T_{K}^{\alpha}=\frac{2 e^{\gamma} D}{\pi} \exp \left(-\frac{2}{|J| \rho_{F}}\right) .
$$

The characteristic energy $T_{K}^{\alpha}$ is reduced significantly compared to the conventional Kondo temperature by factor 2 in the exponential function. This factor appears because the number of channel of the conduction electrons in this case is just half of the normal Kondo system. The resistivity is also calculated as

$$
R=R_{0}\left[1+\frac{\rho_{F}|J|}{2} \log \left(\frac{2 e^{\gamma} D}{\pi k_{B} T}\right)+\cdots\right]
$$

with a factor $1 / 2$.

\section{DISCUSSION}

We investigated the Kondo effect in the presence of spin-orbit coupling. The influence of band splitting was examined by using the Green's function method where we adopted the decoupling scheme to obtain an approximate solution. The Kondo temperature is reduced by the spinorbit interaction, and shows a sudden decrease when $\alpha k_{F}$ is of the order of $k_{B} T_{K}^{0}$. We call this the Kondo collapse due to the spin-orbit coupling. The Kondo effect is suppressed and the $\log T$-dependence of the resistivity will be weakened and concealed. The reduction of $T_{K}$ as a result of the spin-orbit coupling is consistent with the result for the single-impurity Anderson model using the numerical renormalization group technique 13]. In their work the Kondo temperature is a decreasing function of the Rashba energy $E_{R} \propto \alpha^{2}$ when the level of the localized electrons is lowered, that is, in the Kondo region, while it is a increasing function when the localized level is not deep. The variation of the Kondo temperature is approximately linear as a function of $E_{R}$, namely, quadratic in $\alpha$. This is consistent with our result which shows a small variation of the Kondo temperature with the quadratic correction when $\alpha$ is small. In a recent work 12], the Kondo temperature is increased in the presence of the Dzyaloshinski-Moriya interaction. The DzyaloshinskyMoriya interaction, however, vanishes in the Kondo region with particle-hole symmetry $\epsilon_{d}=-U / 2$. Hence the result in ref. 12] seems consistent with the result for the s-d model.

As a limit of strong spin-orbit interaction, we can investigate a crossover to a one-channel Kondo problem. The Kondo problem with the spin-orbit coupling is closely related to a multi-channel Kondo problem. The Kondo temperature is reduced considerably because the degeneracy of the conducting electrons becomes half of the conventional Kondo system in this limit. The specific heat also exhibits a $\log T$-term in the present model with one-channel conduction band, and this term appears in the fifth-order of $\rho J$. This agrees with the original Kondo problem.

The author expresses his sincere thanks to K. Yamaji, I. Hase and J. Kondo for helpful discussion.

\section{APPENDIX}

In this appendix we derive the equation of motion for Green's functions for the single-band s-d model and discuss its physical properties.

\section{A. Green's functions}

$H_{0}$ was diagonalized by $\alpha_{\mathbf{k}}$ and $\beta_{\mathbf{k}}$ :

$$
\begin{aligned}
\alpha_{\mathbf{k}} & =u_{\mathbf{k}} c_{\mathbf{k} \uparrow}+v_{\mathbf{k}} c_{\mathbf{k} \downarrow}, \\
\beta_{\mathbf{k}} & =-v_{\mathbf{k}}^{*} c_{\mathbf{k} \uparrow}+u_{\mathbf{k}} c_{\mathbf{k} \downarrow} .
\end{aligned}
$$


The coefficients $u_{\mathbf{k}}$ and $v_{\mathbf{k}}$ are

$$
u_{\mathbf{k}}=\frac{1}{2}, \quad v_{\mathbf{k}}=-\frac{i k_{x}+k_{y}}{\sqrt{2} k_{\perp}}
$$

satisfying $u_{\mathbf{k}}^{2}+\left|v_{\mathbf{k}}\right|^{2}=1$. The s-d interaction part becomes

$$
\begin{aligned}
H_{s d} & =-\frac{J}{2} \frac{1}{N} \sum_{\mathbf{k k}^{\prime}}\left[S _ { z } \left\{\left(u_{k} u_{k^{\prime}}-v_{k} v_{k^{\prime}}^{*}\right) \alpha_{k}^{\dagger} \alpha_{k^{\prime}}\right.\right. \\
& -\left(u_{k} u_{k^{\prime}}-v_{k}^{*} v_{k^{\prime}}\right) \beta_{\mathbf{k}}^{\dagger} \beta_{\mathbf{k}^{\prime}} \\
& \left.-\left(u_{k} v_{k^{\prime}}+v_{k} u_{k^{\prime}}\right) \alpha_{\mathbf{k}}^{\dagger} \beta_{\mathbf{k}^{\prime}}-\left(u_{k} v_{k^{\prime}}^{*}+v_{k}^{*} u_{k^{\prime}}\right) \beta_{\mathbf{k}^{\dagger}}^{\dagger} \alpha_{\mathbf{k}^{\prime}}\right\} \\
& +S_{+}\left(v_{k} u_{k^{\prime}} \alpha_{\mathbf{k}}^{\dagger} \alpha_{\mathbf{k}^{\prime}}-u_{k} v_{k^{\prime}} \beta_{\mathbf{k}}^{\dagger} \beta_{\mathbf{k}^{\prime}}-v_{k} v_{k^{\prime}} \alpha_{\mathbf{k}}^{\dagger} \beta_{\mathbf{k}^{\prime}}\right. \\
& \left.+u_{k} u_{k^{\prime}} \beta_{\mathbf{k}}^{\dagger} \alpha_{\mathbf{k}^{\prime}}\right) \\
& +S_{-}\left(u_{k} v_{k^{\prime}}^{*} \alpha_{\mathbf{k}}^{\dagger} \alpha_{\mathbf{k}^{\prime}}-v_{k}^{*} u_{k^{\prime}} \beta_{\mathbf{k}}^{\dagger} \beta_{\mathbf{k}^{\prime}}+u_{k} u_{k^{\prime}} \alpha_{\mathbf{k}}^{\dagger} \beta_{\mathbf{k}^{\prime}}\right. \\
& \left.\left.-v_{k}^{*} v_{k^{\prime}} \beta_{\mathbf{k}}^{\dagger} \alpha_{\mathbf{k}^{\prime}}\right)\right] .
\end{aligned}
$$

The single-band s-d model contains only the following s-d interaction,

$$
\begin{aligned}
H_{s d}^{\alpha} & =-\frac{J}{2} \frac{1}{N} \sum_{\mathbf{k k}^{\prime}}\left[S _ { z } \left\{\left(u_{k} u_{k^{\prime}}-v_{k} v_{k^{\prime}}^{*}\right) \alpha_{k}^{\dagger} \alpha_{k^{\prime}}\right.\right. \\
& \left.+S_{+} v_{k} u_{k^{\prime}} \alpha_{k}^{\dagger} \alpha_{k^{\prime}}+S_{-} u_{k} v_{k^{\prime}}^{*} \alpha_{k}^{\dagger} \alpha_{k^{\prime}}\right] .
\end{aligned}
$$

We consider the following Green's functions:

$$
\begin{aligned}
G_{\mathbf{k k}^{\prime}}^{\alpha}(\tau) & =-\left\langle T_{\tau} \alpha_{\mathbf{k}}(\tau) \alpha_{\mathbf{k}^{\prime}}^{\dagger}(0)\right\rangle, \\
\left\langle\left\langle S_{z} \alpha_{\mathbf{k}} ; \alpha_{\mathbf{k}^{\prime}}^{\dagger}\right\rangle\right\rangle_{\tau} & =-\left\langle T_{\tau}\left(S_{z} \alpha_{\mathbf{k}}\right)(\tau) \alpha_{\mathbf{k}^{\prime}}^{\dagger}(0)\right\rangle, \\
\left\langle\left\langle S_{+} \alpha_{\mathbf{k}} ; \alpha_{\mathbf{k}^{\prime}}^{\dagger}\right\rangle\right\rangle_{\tau} & =-\left\langle T_{\tau}\left(S_{+} \alpha_{\mathbf{k}}\right)(\tau) \alpha_{\mathbf{k}^{\prime}}^{\dagger}(0)\right\rangle .
\end{aligned}
$$

The Fourier transforms are defined similarly:

$$
\begin{aligned}
G_{\mathbf{k k}^{\prime}}^{\alpha}(\tau) & =\frac{1}{\beta} \sum_{\omega_{n}} e^{i \omega_{n} \tau} G_{\mathbf{k k}^{\prime}}^{\alpha}\left(i \omega_{n}\right), \\
\left\langle\left\langle S_{z} \alpha_{\mathbf{k}} ; \alpha_{\mathbf{k}^{\prime}}^{\dagger}\right\rangle\right\rangle_{\tau} & =\frac{1}{\beta} \sum_{\omega_{n}} e^{i \omega_{n} \tau}\left\langle\left\langle S_{z} \alpha_{\mathbf{k}} ; \alpha_{\mathbf{k}^{\prime}}^{\dagger}\right\rangle\right\rangle_{\omega}, \\
\left\langle\left\langle S_{+} \alpha_{\mathbf{k}} ; \alpha_{\mathbf{k}^{\prime}}^{\dagger}\right\rangle\right\rangle_{\tau} & =\frac{1}{\beta} \sum_{\omega_{n}} e^{i \omega_{n} \tau}\left\langle\left\langle S_{+} \alpha_{\mathbf{k}} ; \alpha_{\mathbf{k}^{\prime}}^{\dagger}\right\rangle\right\rangle_{\omega} .
\end{aligned}
$$

The equations of motion for these Green's functions are derived as follows.

$$
\begin{aligned}
i \omega_{n} G_{\mathbf{k k}^{\prime}}^{\alpha}\left(i \omega_{n}\right) & =\delta_{\mathbf{k k}^{\prime}}+\xi_{\mathbf{k} \alpha} G_{\mathbf{k k}^{\prime}}^{\alpha}\left(i \omega_{n}\right) \\
& -\frac{J}{2 N} \sum_{q}\left[\left(u_{k} u_{q}-v_{k} v_{q}^{*}\right)\left\langle\left\langle S_{z} \alpha_{\mathbf{q}} ; \alpha_{\mathbf{k}^{\prime}}^{\dagger}\right\rangle\right\rangle_{i \omega_{n}}\right. \\
& +v_{k} u_{q}\left\langle\left\langle S_{+} \alpha_{\mathbf{q}} ; \alpha_{\mathbf{k}^{\prime}}^{\dagger}\right\rangle\right\rangle_{i \omega_{n}} \\
& \left.+u_{k} v_{q}^{*}\left\langle\left\langle S_{-} \alpha_{\mathbf{q}} ; \alpha_{\mathbf{k}^{\prime}}^{\dagger}\right\rangle\right\rangle_{i \omega_{n}}\right],
\end{aligned}
$$

$$
\begin{gathered}
i \omega_{n}\left\langle\left\langle S_{z} \alpha_{\mathbf{k}} ; \alpha_{\mathbf{k}^{\prime}}^{\dagger}\right\rangle\right\rangle_{i \omega_{n}}=\xi_{\mathbf{k} \alpha}\left\langle\left\langle S_{z} \alpha_{\mathbf{k}} ; \alpha_{\mathbf{k}^{\prime}}^{\dagger}\right\rangle\right\rangle_{i \omega_{n}} \\
+\frac{J}{2 N} \sum_{q}\left[-\left(u_{k} u_{q}-v_{k} v_{q}^{*}\right)\left\langle\left\langle S_{z}^{2} \alpha_{\mathbf{q}} ; \alpha_{\mathbf{k}^{\prime}}^{\dagger}\right\rangle\right\rangle_{i \omega_{n}}\right. \\
+v_{k} u_{q}^{*}\left\langle\left\langle S_{+}\left(n_{\mathbf{k} \alpha}-\frac{1}{2}\right) \alpha_{\mathbf{q}} ; \alpha_{\mathbf{k}^{\prime}}^{\dagger}\right\rangle\right\rangle_{i \omega_{n}} \\
\left.-u_{k} v_{q}^{*}\left\langle\left\langle S_{-}\left(n_{\mathbf{k} \alpha}-\frac{1}{2}\right) \alpha_{\mathbf{q}} ; \alpha_{\mathbf{k}^{\prime}}^{\dagger}\right\rangle\right\rangle_{i \omega_{n}}\right] \\
+\frac{J}{2 N} \sum_{\mathbf{q}}\left[-\left(u_{k} u_{q}-v_{k} v_{q}^{*}\right)\left\langle\left\langle S_{+}\left(n_{\mathbf{k} \alpha}-\frac{1}{2}\right) \alpha_{\mathbf{q}^{\prime}} ; \alpha_{\mathbf{k}^{\prime}}^{\dagger}\right\rangle\right\rangle_{i \omega_{n}}\right. \\
\left.+2 u_{k} v_{q}^{*}\left\langle\left\langle S_{z} n_{\mathbf{k} \alpha} \alpha_{\mathbf{q}} ; \alpha_{\mathbf{k}^{\prime}}^{\dagger}\right\rangle\right\rangle_{i \omega_{n}}-u_{k} v_{q}^{*}\left\langle\left\langle S_{+} S_{-} \alpha_{\mathbf{q}} ; \alpha_{\mathbf{k}^{\prime}}^{\dagger}\right\rangle\right\rangle_{i \omega_{n}}\right]
\end{gathered}
$$

$$
\begin{gathered}
i \omega_{n}\left\langle\left\langle S_{-} \alpha_{\mathbf{k}} ; \alpha_{\mathbf{k}^{\prime}}^{\dagger}\right\rangle\right\rangle_{i \omega_{n}}=\xi_{\mathbf{k} \alpha}\left\langle\left\langle S_{+} \alpha_{\mathbf{k}} ; \alpha_{\mathbf{k}^{\prime}}^{\dagger}\right\rangle\right\rangle_{i \omega_{n}} \\
+\frac{J}{2 N} \sum_{\mathbf{q}}\left[\left(u_{k} u_{q}-v_{k} v_{q}^{*}\right)\left\langle\left\langle S_{-}\left(n_{\mathbf{k} \alpha}-\frac{1}{2}\right) \alpha_{\mathbf{q}} ; \alpha_{\mathbf{k}^{\prime}}^{\dagger}\right\rangle\right\rangle_{i \omega_{n}}\right. \\
\left.-2 v_{k} u_{q}\left\langle\left\langle S_{z} n_{\mathbf{k} \alpha} \alpha_{\mathbf{q}} ; \alpha_{\mathbf{k}^{\prime}}^{\dagger}\right\rangle\right\rangle_{i \omega_{n}}-v_{k} u_{q}\left\langle\left\langle S_{-} S_{+} \alpha_{\mathbf{q}} ; \alpha_{\mathbf{k}^{\prime}}^{\dagger}\right\rangle\right\rangle_{i \omega_{n}}\right],
\end{gathered}
$$

where $n_{\mathbf{k} \alpha}=\alpha_{\mathbf{k}}^{\dagger} \alpha_{\mathbf{k}}$. We have unknown functions $\left\langle\left\langle S_{a} n_{\mathbf{k} \alpha} \alpha_{\mathbf{q}} ; \alpha_{\mathbf{k}^{\prime}}^{\dagger}\right\rangle\right\rangle$ for $a=z,+$ and - .

\section{B. Approximate Solution}

To obtain a consistent solution, we adopt the following approximation:

$$
\left\langle\left\langle S_{a} n_{\mathbf{k} \alpha} \alpha_{\mathbf{q}} ; \alpha_{\mathbf{k}^{\prime}}^{\dagger}\right\rangle\right\rangle=\left\langle n_{\mathbf{k} \alpha}\right\rangle\left\langle\left\langle S_{a} \alpha_{\mathbf{q}} ; \alpha_{\mathbf{k}^{\prime}}^{\dagger}\right\rangle\right\rangle .
$$

Using this approximation and the relation $S_{+} S_{-}=3 / 4+$ $S_{z}-S_{z}^{2}$, we obtain

$$
\begin{aligned}
& \left(i \omega_{n}-\xi_{\mathbf{k} \alpha}\right) G_{\mathbf{k k}^{\prime}}^{\alpha}\left(i \omega_{n}\right)=\delta_{\mathbf{k k}^{\prime}} \\
+ & \left(\frac{J}{2 N}\right)^{2} \sum_{\mathbf{q}} \frac{\bar{n}_{\mathbf{q}}-1 / 2}{i \omega_{n}-\xi_{\mathbf{q}}} \sum_{\mathbf{q}^{\prime}}\left[v_{k} u_{q^{\prime}}\left\langle\left\langle S_{+} \alpha_{\mathbf{q}^{\prime}} ; \alpha_{\mathbf{k}^{\prime}}^{\dagger}\right\rangle\right\rangle_{i \omega_{n}}\right. \\
+ & u_{k} v_{q^{\prime}}^{*}\left\langle\left\langle S_{-} \alpha_{\mathbf{q}^{\prime}} ; \alpha_{\mathbf{k}^{\prime}}^{\dagger}\right\rangle\right\rangle_{i \omega_{n}} \\
+ & \left.\left(u_{k} u_{q^{\prime}}-v_{k} v_{q^{\prime}}^{*}\right)\left\langle\left\langle S_{z} \alpha_{\mathbf{q}^{\prime}} ; \alpha_{\mathbf{k}^{\prime}}^{\dagger}\right\rangle\right\rangle_{i \omega_{n}}\right] \\
+ & \frac{3}{8}\left(\frac{J}{2 N}\right)^{2} \sum_{\mathbf{q}} \frac{1}{i \omega_{n}-\xi_{\mathbf{q} \alpha}} \sum_{\mathbf{q}^{\prime}}\left(u_{k} u_{q^{\prime}}+v_{k} v_{q^{\prime}}^{*}\right) G_{\mathbf{q}^{\prime} \mathbf{k}^{\prime}}^{\alpha}\left(i \omega_{n}\right),
\end{aligned}
$$


where $\bar{n}_{\mathbf{q}}=\left\langle n_{\mathbf{q} \alpha}\right\rangle$. Here we define

$$
\begin{aligned}
\Gamma_{\mathbf{k k}^{\prime}}\left(i \omega_{n}\right) & =\sum_{\mathbf{q}}\left[\left(u_{k} u_{q}-v_{k} v_{q}^{*}\right)\left\langle\left\langle S_{z} \alpha_{\mathbf{q}} ; \alpha_{\mathbf{k}^{\prime}}^{\dagger}\right\rangle\right\rangle_{i \omega_{n}}\right. \\
& \left.+v_{k} u_{q}\left\langle\left\langle S_{+} \alpha_{\mathbf{q}} ; \alpha_{\mathbf{k}^{\prime}}^{\dagger}\right\rangle\right\rangle_{i \omega_{n}}+u_{k} v_{q}^{*}\left\langle\left\langle S_{-} \alpha_{\mathbf{q}} ; \alpha_{\mathbf{k}^{\prime}}^{\dagger}\right\rangle\right\rangle_{i \omega_{n}}\right] .
\end{aligned}
$$

This quantity reads after substituting the equations for $\left\langle\left\langle S_{a} \alpha_{\mathbf{q}} ; \alpha_{\mathbf{k}^{\prime}}^{\dagger}\right\rangle\right\rangle$

$$
\begin{aligned}
\Gamma_{\mathbf{k k}^{\prime}} & =\frac{J}{2 N} \sum_{\mathbf{q}} \frac{1}{i \omega_{n}-x i_{\mathbf{k} \alpha}} \sum_{\mathbf{q}^{\prime}}[ \\
& -v_{k} u_{q^{\prime}}\left(\bar{n}_{\mathbf{q}}-\frac{1}{2}\right)\left\langle\left\langle S_{+} \alpha_{\mathbf{q}^{\prime}} ; \alpha_{\mathbf{k}^{\prime}}^{\dagger}\right\rangle\right\rangle_{i \omega_{n}} \\
& -u_{k} v_{q^{\prime}}^{*}\left(\bar{n}_{\mathbf{q}}-\frac{1}{2}\right)\left\langle\left\langle S_{-} \alpha_{\mathbf{q}^{\prime}} ; \alpha_{\mathbf{k}^{\prime}}^{\dagger}\right\rangle\right\rangle_{i \omega_{n}} \\
& -\left(u_{k} u_{q^{\prime}}-v_{k} v_{q^{\prime}}^{*}\right)\left(\bar{n}_{\mathbf{q}}-\frac{1}{2}\right)\left\langle\left\langle S_{z} \alpha_{\mathbf{q}^{\prime}} ; \alpha_{\mathbf{k}^{\prime}}^{\dagger}\right\rangle\right\rangle_{i \omega_{n}} \\
& \left.-\frac{3}{8}\left(u_{k} u_{q^{\prime}}+v_{k} v_{q^{\prime}}^{*}\right) G_{\mathbf{q}^{\prime} \mathbf{k}^{\prime}}^{\alpha}\left(i \omega_{n}\right)\right] \\
& =-\frac{J}{2 N} \frac{3}{8} \sum_{\mathbf{q}} \frac{\bar{n}_{\mathbf{k}}-1 / 2}{i \omega_{n}-\xi_{\mathbf{q} \alpha}} \Gamma_{\mathbf{k} \mathbf{k}^{\prime}}-\frac{J}{2 N} \frac{3}{8} \sum_{\mathbf{q}} \frac{1}{i \omega_{n}-\xi_{\mathbf{q} \alpha}} \\
& \times \sum_{\mathbf{q}^{\prime}}\left(u_{k} u_{q^{\prime}}+v_{k} v_{q^{\prime}}^{*}\right) G_{\mathbf{q}^{\prime} \mathbf{k}^{\prime}}^{\alpha}\left(i \omega_{n}\right) .
\end{aligned}
$$

Then we obtain

$$
\begin{aligned}
G_{\mathbf{k k}^{\prime}}^{\alpha}\left(i \omega_{n}\right) & =\frac{\delta_{\mathbf{k k}^{\prime}}}{i \omega_{n}-\xi_{\mathbf{k} \alpha}}+\frac{3}{8}\left(\frac{J}{2 N}\right)^{2} \frac{1}{i \omega_{n}-\xi_{\mathbf{k} \alpha}} \\
& \times \sum_{\mathbf{q}^{\prime}} \frac{1}{i \omega_{n}-\xi_{\mathbf{q}^{\prime} \alpha}} \frac{1}{1+\frac{J}{2 N} \sum_{\mathbf{p}} \frac{\bar{n}_{\mathbf{p}}-1 / 2}{i \omega_{n}-\xi_{\mathbf{p} \alpha}}} \\
& \times \sum_{\mathbf{q}}\left(u_{k} u_{q}+v_{k} v_{q}^{*}\right) G_{\mathbf{q k}^{\prime}}^{\alpha}\left(i \omega_{n}\right) .
\end{aligned}
$$

We have set $u_{\mathbf{k}}=1 / \sqrt{2}$. Because $v_{\mathbf{k}}$ satisfies $v_{\mathbf{k}}=-v_{-\mathbf{k}}$ and $\left|v_{\mathbf{k}}\right|^{2}=1 / 2$, we have

$$
\begin{aligned}
\sum_{\mathbf{k}} v_{\mathbf{k}}^{*} G_{\mathbf{k k}^{\prime}}^{\alpha}\left(i \omega_{n}\right) & =\frac{v_{\mathbf{k}^{\prime}}^{*}}{i \omega_{n}-\xi_{\mathbf{k}^{\prime} \alpha}}\left[1-\frac{3}{8}\left(\frac{J}{2}\right)^{2} \frac{1}{2} F_{\alpha}\left(i \omega_{n}\right)^{2}\right. \\
& \left.\times \frac{1}{1+(J / 2) G_{\alpha}\left(i \omega_{n}\right)}\right]^{-1}
\end{aligned}
$$

where we set

$$
\begin{aligned}
F_{\alpha}(z) & =\frac{1}{N} \sum_{\mathbf{k}} \frac{1}{z-\xi_{\mathbf{k} \alpha}}, \\
G_{\alpha}(z) & =\frac{1}{N} \sum_{\mathbf{k}} \frac{\bar{n}_{\mathbf{k} \alpha}-1 / 2}{z-\xi_{\mathbf{k} \alpha}} .
\end{aligned}
$$

We define

$$
t(z)=\frac{3 J}{16} \frac{F_{\alpha}(z)}{1+\frac{J}{2} G_{\alpha}(z)-\frac{3}{16}\left(\frac{J}{2}\right)^{2} F_{\alpha}(z)^{2}} .
$$

Then $G_{\mathbf{k k}^{\prime}}^{\alpha}$ and $\Gamma_{\mathbf{k k}^{\prime}}$ read

$$
\begin{aligned}
G_{\mathbf{k k}^{\prime}}^{\alpha}(z) & =\frac{\delta_{\mathbf{k k}^{\prime}}}{z-\xi_{\mathbf{k} \alpha}}+\frac{J}{2 N} \frac{\frac{1}{2}+v_{k} v_{k^{\prime}}^{*}}{\left(z-\xi_{\mathbf{k} \alpha}\right)\left(z-\xi_{\mathbf{k}^{\prime} \alpha}\right)} t(z) \\
\Gamma_{\mathbf{k} \mathbf{k}^{\prime}}(z) & =-\frac{\frac{1}{2}+v_{k} v_{k^{\prime}}^{*}}{z-\xi_{\mathbf{k}^{\prime} \alpha}} t(z)
\end{aligned}
$$

for arbitrary complex number $z$. The Kondo temperature $T_{K}^{\alpha}$ is determined from a singularity of $t(z)$ in the same way as previous sections. We obtain

$$
T_{K}^{\alpha}=\frac{2 e^{\gamma} D}{\pi} \exp \left(-\frac{2}{|J| \rho_{F}}\right) .
$$

The characteristic energy $T_{K}^{\alpha}$ is reduced significantly compared to the conventional Kondo temperature by factor 2 in the exponential function:

$$
T_{K}^{\alpha} \sim\left(\frac{T_{K}^{0}}{D}\right) T_{K}^{0}
$$

This factor appears because the number of channel of the conduction electrons in this case is just half of the normal Kondo system. The resistivity is also calculated as

$$
R=R_{0}\left[1+\frac{\rho_{F}|J|}{2} \log \left(\frac{2 e^{\gamma} D}{\pi k_{B} T}\right)+\cdots\right]
$$

with a factor $1 / 2$.

\section{Entropy and Specific Heat}

The energy expectation value $E=\langle H\rangle$ is given by

$$
\begin{aligned}
E & =\sum_{\mathbf{k}} \xi_{\mathbf{k}}\left\langle\alpha_{\mathbf{k}}^{\dagger} \alpha_{\mathbf{k}}\right\rangle-\frac{J}{2 N} \sum_{\mathbf{k} \mathbf{k}^{\prime}}\left\langle\left\{ S_{z}\left(u_{k} u_{k^{\prime}}-v_{k} v_{k^{\prime}}^{*}\right)\right.\right. \\
& \left.\left.+S_{+} v_{k} u_{k^{\prime}}+S_{-} u_{k} v_{k^{\prime}}^{*}\right\} \alpha_{\mathbf{k}}^{\dagger} \alpha_{\mathbf{k}^{\prime}}\right\rangle \\
& =\frac{1}{\beta} \sum_{\mathbf{k} \omega_{n}} \xi_{\mathbf{k} \alpha} G_{\mathbf{k} \mathbf{k}}^{\dagger}\left(i \omega_{n}\right)-\frac{J}{2} \frac{1}{\beta N} \sum_{\mathbf{k}} \Gamma_{\mathbf{k k}}\left(i \omega_{n}\right) \\
& =\frac{1}{\beta} \sum_{\mathbf{k} \omega_{n}} \frac{\xi_{\mathbf{k} \alpha}}{i \omega_{n}-\xi_{\mathbf{k} \alpha}}+\frac{J}{2} \frac{1}{\beta N} \sum_{\mathbf{k} \omega_{n}} \frac{i \omega_{n} t\left(i \omega_{n}\right)}{\left(i \omega_{n}-\xi_{\mathbf{k} \alpha}\right)^{2}}
\end{aligned}
$$

The expectation value of the interaction Hamiltonian is denoted as $V . V$ is given by

$$
\begin{aligned}
V & =-\frac{J}{2 N} \sum_{\mathbf{k k}^{\prime}}\left\langle\left\{ S_{z}\left(u_{k} u_{k^{\prime}}-v_{k} v_{k^{\prime}}^{*}\right)+S_{+} v_{k} u_{k^{\prime}}\right.\right. \\
& \left.\left.+S_{-} u_{k} v_{k^{\prime}}^{*}\right\} \alpha_{\mathbf{k}}^{\dagger} \alpha_{\mathbf{k}^{\prime}}\right\rangle \\
& =-\frac{J}{2} \frac{1}{\beta N} \sum_{\mathbf{k}} \Gamma_{\mathbf{k k}}\left(i \omega_{n}\right)=\frac{J}{2} \frac{1}{\beta N} \sum_{\mathbf{k} \omega_{n}} \frac{t\left(i \omega_{n}\right)}{i \omega_{n}-\xi_{\mathbf{k} \alpha}}
\end{aligned}
$$


This is written as

$$
V=\frac{J}{2} \rho(0) \operatorname{Re} \int_{-D}^{D} d \omega f(\omega) t(\omega-i \delta),
$$

where we adopted the approximation

$$
F_{\alpha}(\omega \pm i \delta)=\mp \pi \rho(0) i
$$

$\rho(\omega)$ is the density of states of conduction electrons.

We need $t(z)$ to estimate $V . G_{\alpha}(z)$, which appears in the denominator of $t(z)$, contains a singularity. $G_{\alpha}(z)$ is written as

$$
G_{\alpha}(z)=R_{\alpha}(z)+\frac{J}{2 N} \frac{1}{\beta N} \sum_{\mathbf{k} \omega_{n}} \frac{1}{z-\xi_{\mathbf{k} \alpha}} \frac{t\left(i \omega_{n}\right)}{\left(i \omega_{n}-\xi_{\mathbf{k} \alpha}\right)^{2}}
$$

where

$$
R_{\alpha}(z)=\frac{1}{\beta} \sum_{\omega_{n}} \frac{F_{\alpha}\left(i \omega_{n}\right)-F_{\alpha}(z)}{z-i \omega_{n}}-\frac{1}{2} F_{\alpha}(z) .
$$

$R_{\alpha}(z)$ is evaluated as 18

$$
R_{\alpha}(\omega-i \delta) \approx \rho(0)\left[\psi\left(\frac{1}{2}+\frac{\beta D}{2 \pi}\right)-\psi\left(\frac{1}{2}+i \frac{\beta z}{2 \pi}\right)\right],
$$

where $\psi$ is the digamma function and $D$ is the cutoff energy. We use the following relation,

$$
\begin{aligned}
& 1+\frac{\rho(0) J}{2}\left[\log \left(\frac{D}{2 \pi k_{B} T}\right)-\psi\left(\frac{1}{2}+i \frac{\beta \omega}{2 \pi}\right)\right] \\
= & \frac{\rho(0) J}{2}\left[\log \frac{T_{K}^{\alpha}}{T}-g(\beta \omega)\right],
\end{aligned}
$$

where

$$
g(x)=\psi\left(\frac{1}{2}+i \frac{x}{2 \pi}\right)-\psi\left(\frac{1}{2}\right)
$$

Then the interaction energy is

$$
V=-\frac{3 \pi}{16} \rho(0) J \operatorname{Im} \int_{-D}^{D} d \omega f(\omega) \frac{1}{\log \left(T_{K}^{\alpha} / T\right)-g(\beta \omega)}
$$

where we neglected the term of the order of $(\rho(0) J)^{2}$ in the denominator of $t(z)$. $V$ has a logarithmic temperature dependence. Because of the relation between the free energy and $V$,

$$
V=J \frac{\partial F}{\partial J}
$$

the additional entropy $\Delta S(T)$ is

$$
\Delta S(T)=-\frac{\partial}{\partial T}\left(F-F_{0}\right)=-\int_{0}^{J} \frac{d J^{\prime}}{J^{\prime}} \frac{\partial}{\partial T} V\left(J^{\prime}, T\right)
$$

To estimate $V$, we use the expansion formula for the Fermi distribution function $f(\omega)$ :

$$
\int_{-D}^{D} d \omega f(\omega) h(\omega)=\int_{-D}^{0} d \omega h(\omega)+\frac{\pi^{2}}{6}\left(k_{B} T\right)^{2} h^{\prime}(0),
$$

for a differentiable function $h(\omega)$. Using this, we obtain

$$
\begin{aligned}
V & =-\frac{3 \pi}{16} \rho(0) J \operatorname{Im} \int_{-D}^{0} d \omega \frac{1}{\log \left(T_{K}^{\alpha} / T\right)-g(\beta \omega)} \\
& -\left.\frac{3 \pi}{16} \frac{\pi^{2}}{6}\left(k_{B} T\right)^{2} \rho(0) J \operatorname{Im} \frac{\partial}{\partial \omega} \frac{1}{\log \left(T_{K}^{\alpha} / T\right)-g(\beta \omega)}\right|_{\omega=0} .
\end{aligned}
$$

We are interested in logarithmic terms $\log \left(D / k_{B} T\right)$, $\log \left(D / k_{B} T\right)^{2}$ and so on in the region $\left|\log \left(T_{K}^{\alpha} / T\right)\right| \gg 1$. The second term is written as

$$
V_{2}=-\frac{\pi^{4}}{128} k_{B} T \rho(0) J \frac{1}{\left(\log \left(T_{K}^{\alpha} / T\right)\right)^{2}} .
$$

This is expanded as in terms of $\rho(0) \mathrm{J}$ :

$$
\begin{aligned}
V_{2} & =\frac{\pi^{4}}{32}\left(\frac{\rho(0) J}{2}\right)^{4} k_{B} T \log \left(\frac{D}{k_{B} T}\right) \\
& -\frac{3 \pi^{4}}{64}\left(\frac{\rho(0) J}{2}\right)^{5} k_{B} T \log \left(\frac{D}{k_{B} T}\right)^{2} .
\end{aligned}
$$

In the first term of $V$, the logarithmic corrections never emerge from the region where $\beta \omega$ is large because we have $g(\beta \omega) \sim \log (\beta \omega)$ for large $\omega$ and the $T$-dependence is canceled with $\log \left(T_{K}^{\alpha} / T\right)$. When $\beta \omega$ is small, $g(\beta \omega)$ is expressed in a power series of $\beta \omega$. A dominant contribution is of the order of $\left(\log \left(T_{K}^{\alpha} / T\right)\right)^{-2}$. The integral is restricted on the interval $\left(-k_{B} T, 0\right)$ and the first term $V_{1}$ is estimated as

$$
\begin{aligned}
V_{1} & \simeq-\frac{3 \pi}{16} \rho(0) J \frac{1}{\left(\log \left(T_{K}^{\alpha} / T\right)\right)^{2}} \int_{-k_{B} T}^{0} d \omega \operatorname{Im} \psi\left(\frac{1}{2}+i \frac{\beta \omega}{2 \pi}\right) \\
& =-\frac{3 \pi}{16} k_{B} T \rho(0) J \frac{1}{\left(\log \left(T_{K}^{\alpha} / T\right)\right)^{2}} \pi\left[-\frac{1}{8}+0.0052\right. \\
& -0.00738+0.000026 \cdots] .
\end{aligned}
$$

As a result, $V$ is given as

$$
V=-\frac{A}{2} k_{B} T \rho(0) J \frac{1}{\left(\log \left(T_{K}^{\alpha} / T\right)\right)^{2}},
$$

for a constant $A>0$.

From the relation $T_{K}^{\alpha}=D \exp (2 /(\rho(0) J))$, we have

$$
\frac{d \rho(0) J}{\rho(0) J}=-\frac{1}{\log \left(T_{K}^{\alpha} / D\right)} d \log T_{K}^{\alpha} .
$$

Using this formula, the entropy obtained from the interaction energy $V$ is

$$
\Delta S=-\frac{\partial \Delta F}{\partial T},
$$


where

$$
\begin{aligned}
\Delta F & =-k_{B} A\left[T \frac { 1 } { ( \operatorname { l o g } ( D / T ) ) ^ { 2 } } \left(\frac{\rho(0) J}{2}+\frac{1}{\log \left(T_{K}^{\alpha} / T\right)}\right.\right. \\
& \left.\left.-\frac{2}{\log (D / T)} \log \left|\frac{\rho(0) J}{2} \log \left(\frac{T_{K}^{\alpha}}{T}\right)\right|\right)\right],
\end{aligned}
$$

is the free energy. Because of the relation

$$
\log \left(\frac{T_{K}^{\alpha}}{T}\right)=\frac{2}{\rho(0) J}+\log \left(\frac{2 e^{\gamma}}{\pi} \frac{D}{k_{B} T}\right)
$$

up to the fifth order of $\rho(0) J, \Delta S$ is given as

$$
\begin{aligned}
\Delta S & =k_{B} A\left[\frac{1}{3}\left(\frac{\rho(0) J}{2}\right)^{3}+\frac{1}{2}\left(\frac{\rho(0) J}{2}\right)^{4}\right. \\
& -\frac{1}{2}\left(\frac{\rho(0) J}{2}\right)^{4} \log \left(\frac{D}{k_{B} T}\right)+\frac{3}{5}\left(\frac{\rho(0) J}{2}\right)^{5}\left(\log \frac{D}{k_{B} T}\right) \\
& \left.-\frac{6}{5}\left(\frac{\rho(0) J}{2}\right)^{5} \log \left(\frac{D}{k_{B} T}\right)\right] .
\end{aligned}
$$

The logarithmic term first appears in the fourth order of $\rho(0) J$. Then the correction to the specific heat $\Delta C=$ $T \partial \Delta S / \partial T$ is

$$
\frac{\Delta C}{k_{B}} \simeq \frac{A}{2}\left(\frac{\rho(0) J}{2}\right)^{4}\left[1-\frac{12}{5}\left(\frac{\rho(0) J}{2}\right) \log \left(\frac{D}{k_{B} T}\right)\right] .
$$

Hence the specific heat exhibits a logarithmic behavior at low temperatures. A $\log T$-term appears in the fifth order of $\rho(0) J$; this agrees with the original Kondo problem [2]. In the original Kondo problem, the entropy and the specific heat were evaluated as [2, 19]

$$
\begin{aligned}
& \Delta S_{s d} \simeq k_{B} \frac{\pi^{2}}{4}(\rho J)^{3}\left[1-3 \rho J \log \left(\frac{D}{k_{B} T}\right)\right] \\
& \Delta C_{s d} \simeq k_{B} \frac{3 \pi^{3}}{4}(\rho J)^{4}\left[1-4 \rho J \log \left(\frac{D}{k_{B} T}\right)\right]
\end{aligned}
$$

This suggests that 18

$$
\begin{aligned}
\Delta C_{s d} & \simeq k_{B} \frac{3 \pi^{3}}{4}(\rho J)^{4} \frac{1}{\left(1+\rho J \log \left(D / k_{B} T\right)^{4}\right.} \\
& \simeq k_{B} \frac{3 \pi^{3}}{4} \frac{1}{\left(\log \left(T_{K} / T\right)\right)^{4}},
\end{aligned}
$$

as an expansion in terms of $1 / \log \left(T_{K} / T\right)$. In the present model, the coefficients are reduced, where 4 is reduced to $12 / 5$ in front of $\rho J \log \left(D / k_{B} T\right)$ in the specific heat compared to the usual s-d model, and the divergence near the Kondo temperature is moderated. Because the formation of a local singlet by the the conduction electrons is weakened in a one-channel case, the entropy decreases more slowly as the temperature is decreased.

In the region $\left|\log \left(D / k_{B} T\right)\right| \gg 1$ and $\left|\log \left(T_{K}^{\alpha} / T\right)\right| \gg$ $1, \Delta S$ is obtained as a double-power series of $1 / \log \left(D / k_{B} T\right)$ and $1 / \log \left(T_{K}^{\alpha} / T\right)$ :

$$
\Delta S \simeq k_{B} A\left[\frac{1}{\left(\log \left(D / k_{B} T\right)\right)^{2}}\left(\frac{\rho(0) J}{2}+\frac{1}{\log \left(T_{K}^{\alpha} / T\right)}\right)\right]
$$

Then we obtain

$$
\Delta C \simeq k_{B} A \frac{1}{\left(\log \left(D / k_{B} T\right)\right)^{2}} \frac{1}{\left(\log \left(T_{K}^{\alpha} / T\right)\right)^{2}}
$$

[1] J. Kondo: Prog. Theor. Phys. 32 (1964) 37.

[2] J. Kondo: Solid State Physics 23 (1969) 183.

[3] E. Bauer, G. Hilshcer, H. Michor, Ch. Paus, E. W. Sceidt, A. Gribanov, Yu. Seropegin, H. Noel, M. Sigrist, and P. Rogl: Phys. Rev. Lett. 92 (2004) 027003.

[4] H. Q. Yuan, D. F. Agterberg, N. Hayashi, P. Badica, D. Vandervelde, K. Togano, M. Sigrist and M. B. Salamon: Phys. Rev. Lett. 97 (2006) 017006.

[5] M. Nishiyama, Y. Inada and G. Q. Zheng: Phys. Rev. Lett. 98 (2007) 047002.

[6] N. Kimura, K. Ito, H. Aoki, S. Uji and T. Terashima: Phys. Rev. Lett. 98 (2007) 197001.

[7] M. A. Measson, H. Muranaka, T. Kawai, Y. Ota, K. Sugiyama, M. Hagiwara, K. Kindo, T. Takeuchi, K. Shimizu, F. Honda, R. Settai and Y. Onuki: J. Phys. Soc. Jpn. 78 (2009) 124713.

[8] F. Honda, I. Bonalde, K. Shimizu, S. Yoshiuchi, Y. Hi- rose, T. Nakamura, R. Settai and Y. Onuki: Phys. Rev. B81 (2010) 140507.

[9] A. D. Hillier, J. Quintanilla and R. Cywinski: Phys. Rev. Lett. 102 (2009) 117007.

[10] K. Samokhin, E. S. Zijlstra and S. K. Bose: Phys. Rev. B69 (2004) 094514.

[11] I. Hase and T. Yanagisawa: J. Phys. Soc. Jpn. 78 (2009) 084724.

[12] M. Zarea, S. E. Ulloa and N. Sandler: Phys. Rev. Lett. 108 (2012) 046601.

[13] R. Zitko and J. Bonca: Phys. Rev B84 (2011) 193411.

[14] X. Y. Feng and F.-C. Zhang: J. Phys. Condens. Matter 23 (2011) 105602.

[15] D. Zubarev: Sov. Phys. Uspekhi 3 (1960) 320; Nonequilibrium Statistical Thermodynamics, Plenum Pub. Corp. (1974).

[16] Y. Nagaoka: Phys. Rev. 138 (1965) A1112. 
[17] D. R. Hamann: Phys. Rev. D158 (1967) 570.

[18] J. Zittartz and E. Müller-Hartmann: Z. Phys. 212 (1968) 380.
[19] K. Yosida and H. Miwa: Prog. Theor. Phys. 41 (1969) 1416. 